\title{
Evaluation of Tuner-Based Noise-Parameter Extraction Methods for Very Low Noise Amplifiers
}

\author{
Leonid Belostotski, Member, IEEE, and James W. Haslett, Fellow, IEEE
}

\begin{abstract}
This paper compares the performance of source-tuner noise-parameter extraction methods used to measure noise parameters of low-noise amplifiers that have very low $(<1 \mathrm{~dB})$ noise figures. The methods discussed are known as the Cold method and the modified $Y$-factor method (or Hot-Cold method). The paper describes equations used in the extraction algorithms. In a Monte Carlo analysis by randomly adding various sources of uncertainties to "measurements," created with a computer simulation, performances of the noise parameter extraction methods are compared. It is shown that the iterative Cold method and the direct Cold method are the best at extracting $R_{n}$ and $\Gamma_{\text {opt }}$ noise parameters in terms of lowest standard deviation and close proximity of the extracted mean values to the true values. The simplified Cold method, used in a number of commercial systems, has largest systematic offsets in extracted noise parameters while being the quickest to perform. The modified $Y$-factor method is the slowest to perform due to additional time required for hot measurements. This method is marginally the most accurate to extract $F_{\min }$. These conclusions are also supported with measurement results. This study assembles in one place necessary theoretical background information to serve as a reference for those who are working in the field of noise parameter extraction using tuner-based methods.
\end{abstract}

Index Terms-Cold noise measurement, hot-cold noise measurement, noise parameters, noise source, measurement uncertainty, $Y$-factor.

\section{INTRODUCTION}

$\mathbf{V}$ ARIOUS aspects of noise-parameter measurements of two-port networks have been discussed in [1]-[21]. In this study, we investigate systematic offsets and standard deviations in the measurements performed with tuner-based noise parameter extraction methods. The motivation for this study is to estimate errors in tuner-based noise-parameter extraction systems that are intended for evaluation of very low-noise amplifiers (LNAs), such as an LNA discussed in this paper that was designed for the Square Kilometre Array (SKA) radio telescope [22]-[24].

Today's LNAs routinely attain noise figures below $1 \mathrm{~dB}$, some of which are reported in [25]-[35], and require precision

Manuscript received June 18, 2009. First published December 18, 2009; current version published January 13, 2010. This work was supported by the Natural Sciences and Engineering Research Council of Canada, the Alberta Ingenuity New Faculty Program, the Alberta Provincial Government under the iCORE Program, the National Research Council's Dominion Radio Astrophysical Observatory, and CMC Microsystems.

The authors are with the Department of Electrical and Computer Engineering, University of Calgary, Calgary, AB, Canada T2N $1 \mathrm{~N} 4$.

Color versions of one or more of the figures in this paper are available online at http://ieeexplore.ieee.org.

Digital Object Identifier 10.1109/TMTT.2009.2036411

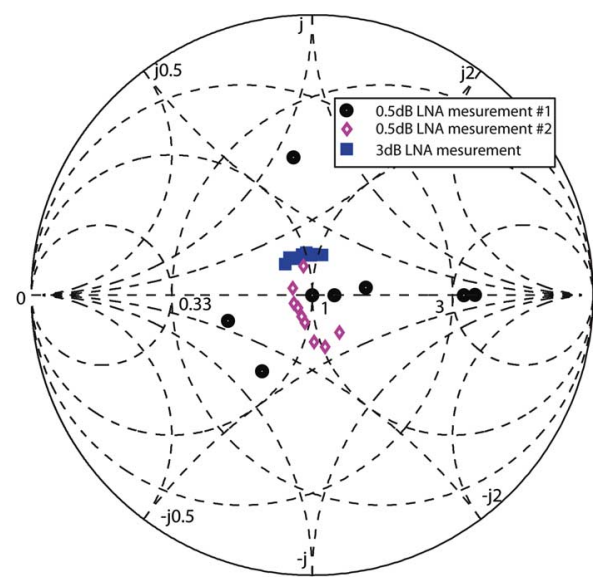

Fig. 1. Measurements of optimum signal-source reflection coefficients for minimum noise of a 3-dB noise figure LNA follow an expected locus. Measurements of optimum signal-source reflection coefficients for minimum noise of a 0.5-dB noise figure LNA show large scatter. The measurements were performed with commercial tuner-based noise-parameter extraction systems over an $800-\mathrm{MHz}-1.4-\mathrm{GHz}$ frequency range. While the commercial algorithms used to measure the 0.5-dB LNA are proprietary, simulations in Section III suggest that the tuner repeatability, impedance constellation selection, and insufficient averaging are likely causes of the data scatter.

noise measurement equipment for full characterization. While commercial parameter extraction systems are acceptable for measuring LNAs with noise figures $>1 \mathrm{~dB}$, their performance may suffer from measurement errors for very low-noise amplifiers, as demonstrated in Fig. 1, where optimum signal-source reflection coefficients for minimum noise of two amplifiers measured with commercial noise parameter extraction systems are shown.

Tuner-based measurements are subdivided into two methods: the Cold method [1]-[8], and the modified $Y$-method, also known as the tuner-based Hot-Cold method [8]-[11]. Other methods of measuring noise parameters exist. Some are based on the representation of the noise signals in terms of noise wave amplitudes [12]-[18]. Another set of noise parameter extraction techniques in [19]-[21] is based on a single noise figure measurement and fitting the result to a device-under-test (DUT) noise model determined analytically or experimentally by using other techniques. The latter two measurement methods are beyond the intended scope of this paper. This work discusses the theory of tuner-based noise-parameter extraction and presents both a Monte Carlo and an experimental analysis of noise parameter extraction methods. Appendix I contains a list of symbols and description of notations for the readers' reference. 


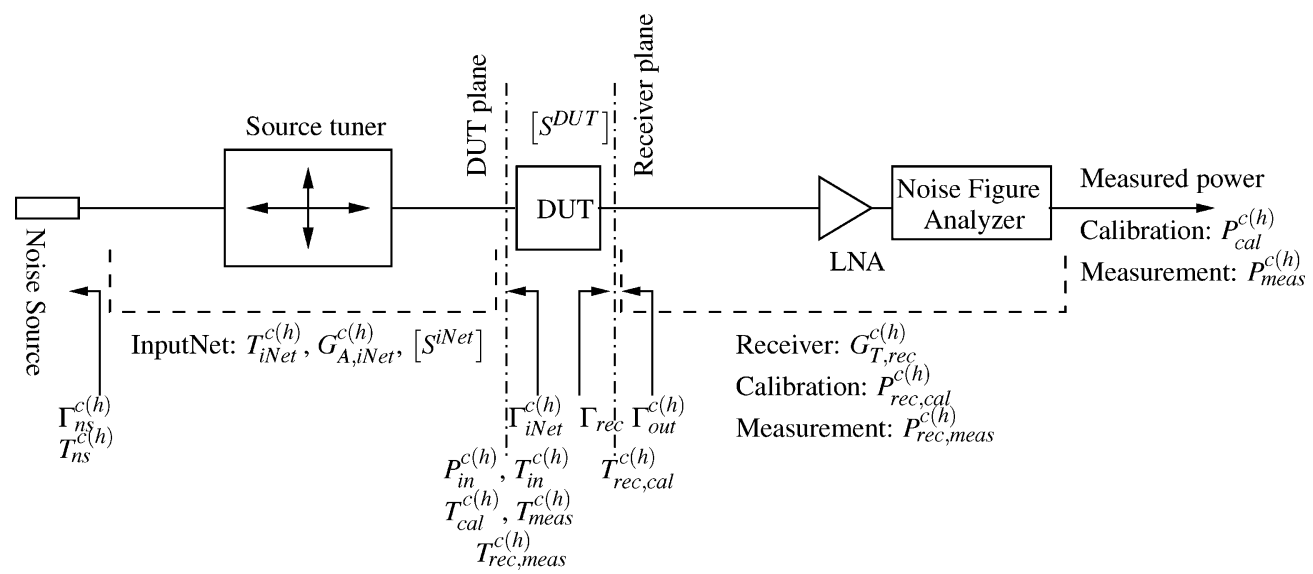

Fig. 2. Cold measurement system and modified $Y$-factor measurement system. For brevity, measurements that depend on whether the noise source is ofF or ON are indicated by the superscript $c(h)$.

\section{NoISE MEASurement Methods}

The most standard method of measuring noise figure is known as the $Y$-method and it is used in all noise figure analyzers [36], [37]. This method is conceptually the simplest, but its accuracy is poor and this method does not allow for noise parameter extraction, and therefore, is not discussed in this paper.

The extraction of the noise parameters $\left(F_{\min }, R_{n}\right.$, and $\left.\Gamma_{\mathrm{opt}}\right)$ requires measurements of the DUT performance when driven by a signal source with varying impedance. These measurements are covered in Sections II-A-C where equations required for noise parameter extraction from noise power measurements are discussed.

\section{A. Temperature Effects}

Modern noise figure analyzers such as the N8975A with noise sources such as the $\mathrm{N} 4000 \mathrm{~A}$ report Cold noise temperature ${ }^{1} T_{\mathrm{ns}}^{c}$, and therefore, $T_{\mathrm{ns}}^{c}$, is available for all calculations discussed in this paper. The noise source Hot noise temperature ${ }^{2} T_{\mathrm{ns}}^{h}$ is determined from the excess noise ratio (ENR) supplied by the noise source manufacturer, defined as [5], [36]

$$
E=\frac{T_{\mathrm{ns}}^{h}\left(1-\left|\Gamma_{\mathrm{ns}}^{h}\right|^{2}\right)-T_{\mathrm{ns}}^{c}}{T_{0}}
$$

where the term in brackets converts noise temperature $T_{\mathrm{ns}}^{h}$ to the corresponding effective noise temperature, ${ }^{3}$ and $\Gamma_{\mathrm{ns}}^{h}$ is the noise source reflection coefficient in the Hot state.

The noise factor (figure) of a DUT is defined as the ratio of the available signal-to-noise ratio at the circuit input to the available signal-to-noise ratio at the circuit output [38]. An equivalent definition in terms of noise temperatures at the ambient room temperature of $T_{a}$ can be written as

$$
\begin{aligned}
F_{\mathrm{DUT}}\left(T_{a}\right) & =1+\frac{T_{\mathrm{DUT}}\left(T_{a}\right)}{T_{0}} \\
& =1+\frac{T_{\mathrm{DUT}}\left(T_{0}\right)}{T_{0}} \frac{T_{a}}{T_{0}}
\end{aligned}
$$

${ }^{1}$ Noise temperatures refer to the temperatures that yield the available noise power from a source.

${ }^{2}$ The value of $T_{\mathrm{ns}}^{h}$ found from (1) is only correct when $T_{\mathrm{ns}}^{c}=T_{0}$ [36]. When $T_{\mathrm{ns}}^{c} \neq T_{0}, T_{\mathrm{ns}}^{h}$ needs to be corrected accordingly [36], [37].

${ }^{3}$ The effective noise temperatures yield the power emerging from a port. where $T_{0}=290 \mathrm{~K}$ is the reference temperature, $T_{\mathrm{DUT}}\left(T_{0}\right)$ is the effective input noise temperature of a DUT at $T_{0}$, and $T_{\text {DUT }}\left(T_{a}\right)$ is the effective input noise temperature of a DUT and is a function of the ambient room temperature $T_{a}$. In this study, we are interested in $F_{\mathrm{DUT}}\left(T_{0}\right)=1+T_{\mathrm{DUT}}\left(T_{0}\right) / T_{0}$, which can be obtained from measured $F_{\mathrm{DUT}}\left(T_{a}\right)$ by using (3). The mechanical-tuner-based noise parameter extraction takes a significant amount of time. During this time, the ambient temperature may experience small variations, which may result in measurement errors. Expression (3) accounts for this where it is assumed that the noise power of a DUT and of a noise power measuring device (receiver) vary with temperature. While the noise figure analyzer might have internal temperature stabilization, an amplifier, cables, isolators, and other external components located between the DUT and noise figure analyzer that define the receiver noise figure are exposed to the ambient temperature. Although the amplifier and the DUT noise temperatures may not scale exactly linearly with ambient temperature, a linear approximation is accurate for small changes around the ambient and is used in this study. Transistors such as MOSFETs can be modeled to a first order with linearly temperature-dependent drain- and gate-noise currents [39]-[43] over a small temperature range. Noise temperatures of LNAs based on bipolar transistors scale nearly linearly with ambient temperature [30], particularly over a small temperature range. Note that $F_{\text {DUT }}$ in (3) remains referenced to $T_{0}$ to conform with the standard noise factor definition [38], [44].

\section{B. Cold Method}

The Cold method uses source impedance tuners to generate varying signal-source reflection coefficients at the DUT input [1]-[8]. The block diagram of the Cold method is shown in Fig. 2. The measurement procedure is broken into the following five steps. Step 1 is performed to collect required $S$-parameter information. Steps 2-4 are the receiver calibration steps. During Step 2, Hot-Cold measurements are performed. Step 3 describes the procedure to determine the tuner noise parameters. This is followed by Step 4 that uses measured data from Step 2 and is needed to complete the extraction of the receiver gain. This gain is used during DUT measurements described in Step 5.

Step 1: S-Parameter Measurement: The system calibration and noise parameter measurements with the Cold-method based 
system require measurements of: 1) the $S$-parameters [ $\left.S^{i \text { Net}}\right]$ of the network located between the noise source and the DUT plane, called InputNet in this paper, for each tuner setting used during noise parameter measurements; 2) the input reflection coefficient of the receiver ${ }^{4} \Gamma_{\text {rec }}$ at the receiver plane; 3) $S$-parameters of the DUT $\left[S^{\mathrm{DUT}}\right]$; and 4) OFF and ON reflection coefficients of the noise source $\Gamma_{\mathrm{ns}}^{c(h)}$. These electrical measurements are performed with a vector network analyzer (VNA), not shown in Fig. 2.

Step 2: Hot-Cold Measurement for Receiver Gain Determination: The DUT in Fig. 2 is replaced by a "THRU," effectively connecting the receiver plane to the DUT plane, and the initial noise power measurements required for receiver noise parameters and receiver gain determination are obtained. To measure the receiver noise parameters and receiver gain, first the noise source is toggled from OFF to ON and two noise powers $P_{\text {cal }}^{c(h)}$ are reported by the noise figure analyzer. These two readings are related to the receiver transducer gain $G_{T \text {,rec }}^{c(h)}$ and noise power by

$$
\left\{\begin{array}{l}
P_{\mathrm{cal}}^{c}=\left(P_{\mathrm{in}}^{c}+\frac{T_{a, 0}^{c}}{T_{0}} P_{\mathrm{rec}, \mathrm{cal}}^{c}\right) G_{T, \mathrm{rec}}^{c} \\
P_{\mathrm{cal}}^{h}=\left(P_{\mathrm{in}}^{h}+\frac{T_{a, 0}^{h}}{T_{0}} P_{\mathrm{rec}, \mathrm{cal}}^{h}\right) G_{T, \mathrm{rec}}^{h}
\end{array}\right.
$$

where $P_{\text {in }}^{c(h)}$ are known and are the available noise powers from the noise source and InputNet at the receiver input, $T_{a, 0}^{c(h)}$ are measured ambient temperatures, $P_{\text {rec,cal }}^{c(h)}$ are the receiver-plane referred noise powers of the receiver at $T_{0}$, and $G_{T \text {,rec }}^{c(h)}$ are transducer gains that can be expressed in the familiar form as [45]

$$
G_{T, \mathrm{rec}}^{c(h)}=\underbrace{\frac{1-\left|\Gamma_{i \mathrm{Net}}^{c(h)}\right|^{2}}{\left|1-\Gamma_{i \mathrm{Net}}^{c(h)} \Gamma_{\mathrm{rec}}\right|^{2}}}_{M^{c(h)}} \underbrace{\left|S_{21}^{\mathrm{rec}}\right|^{2} \frac{1-\left|\Gamma_{L}^{\mathrm{rec}}\right|^{2}}{\left|1-\Gamma_{L}^{\mathrm{rec}} S_{22}^{\mathrm{rec}}\right|^{2}}}_{G_{\mathrm{rec}}} .
$$

In (5), an unknown internal receiver load is represented by $\Gamma_{L}^{\text {rec }}$, unknown internal receiver $S$-parameters are represented by $\left[S^{\text {rec}}\right]$, and $M^{c(h)}$ represent measurable input mismatch terms. Unknown and individually unmeasurable $\Gamma_{L}^{\text {rec }}$ and $\left[S^{\text {rec }}\right]$ are grouped together into $G_{\mathrm{rec}}$ in (5) and $G_{\mathrm{rec}}$ will be found in Step 4. Expressions for $P_{\text {in }}^{c(h)}$ used in (4) are obtained from

$$
P_{\mathrm{in}}^{c(h)}=k B \underbrace{\left[T_{\mathrm{ns}}^{c(h)}+T_{i \mathrm{Net}}^{c(h)}\right] G_{A, i \mathrm{Net}}^{c(h)}}_{T_{\mathrm{in}}^{c(h)}}
$$

where $T_{i \text { Net }}^{c(h)}$ are the noise temperatures of InputNet in Fig. 2 referred to the noise source plane [for derivation see (B.5)] and $G_{A, i \text { Net }}^{c(h)}$ are the available gains of InputNet calculated from measured $S$-parameters $\left[S^{i N e t}\right]$, as shown in (A.3). $T_{\mathrm{ns}}^{c(h)}+T_{i \mathrm{Net}}^{c(h)}$ represent total noise temperatures at the noise source plane and when multiplied by the available gain $G_{A, i \mathrm{Net}}^{c(h)}$ give the noise temperatures of the noise source and InputNet at

\footnotetext{
${ }^{4}$ For receivers that may automatically update their RF attenuation settings based on strengths of input signals, the input reflection coefficient measurements and all receiver noise calibration described in this paper must be performed with the RF attenuation fixed to the level that the receiver selects during DUT measurements.
}

the DUT plane in Fig. 2, represented by $T_{\text {in }}^{c(h)}$. Rewriting (4) in terms of $M^{c(h)}$ and $G_{\text {rec }}$ defined in (5) gives

$$
\left\{\begin{array}{l}
P_{\mathrm{cal}}^{c}=\left(P_{\mathrm{in}}^{c}+\frac{T_{a, 0}^{c}}{T_{0}} P_{\mathrm{rec}, \mathrm{cal}}^{c}\right) M^{c} G_{\mathrm{rec}} \\
P_{\mathrm{cal}}^{h}=\left(P_{\mathrm{in}}^{h}+\frac{T_{a, 0}^{h}}{T_{0}} P_{\mathrm{rec}, \mathrm{cal}}^{h}\right) M^{h} G_{\mathrm{rec}}
\end{array}\right.
$$

which relates directly measured quantities $P_{\text {cal }}^{c(h)}$ and $T_{a, 0}^{c(h)}$ to indirectly measured $P_{\text {in }}^{c(h)}$ from (6) and $M^{c(h)}$ from (5) and to unknown $P_{\text {rec,cal }}^{c(h)}$ and $G_{\text {rec }}$. An evaluation of $G_{\text {rec }}$ is based on (7). However, to complete the determination of $G_{\text {rec }}$, one needs to determine $P_{\text {rec,cal }}^{c(h)}$, which will be discussed in Steps 3 and 4.

Step 3: Receiver Calibration: The receiver calibration proceeds with the noise source turned OFF and the tuner manipulated to present $N(N \geq 4)$ different signal-source impedances to the noise figure analyzer input. For each such impedance $i, 1 \leq i \leq N$, the noise power $P_{\mathrm{cal}, i}^{c}$ and the ambient temperature $T_{a, i}^{c}$ are measured. These two relate to the receiver-plane referred receiver noise power $P_{\text {rec,cal }, i}^{c}$ and receiver transducer gain $G_{T, \text { rec }, i}^{c}$ by

$$
P_{\mathrm{cal}, i}^{c}=\left\{P_{\mathrm{in}, i}^{c}+\frac{T_{a, i}^{c}}{T_{0}} P_{\mathrm{rec}, \mathrm{cal}, i}^{c}\right\} G_{T, \mathrm{rec}, i}^{c}
$$

which, in turn, are related to the noise factors of the receiver $F_{\text {rec }, i}$ by

$$
F_{\mathrm{rec}, i}=1+\frac{P_{\mathrm{cal}, i}^{c}}{k B G_{T, \mathrm{rec}, i}^{c} T_{a, i}^{c}}-\frac{P_{\mathrm{in}, i}^{c}}{k B T_{a, i}^{c}} .
$$

In (9), $P_{\mathrm{in}, i}^{c}$ is determined from (6) and is the noise power available at the DUT plane connected with the "THRU" to the receiver input for the $i$ th signal-source impedance.

When a sufficient number of $P_{\mathrm{cal}, i}^{c}$ are measured, the noise parameter extraction can proceed once $G_{T, \text { rec }}^{c}=M^{c} G_{\text {rec }}$ is obtained, as described below in Step 4. The parameter extraction can be performed with a number of extraction algorithms available [46]-[49] that fit the calculated $F_{\text {rec }, i}$ to a general expression of the noise factor of a device [45]

$$
F=F_{\min }+\frac{4 R_{n}}{Z_{0}} \frac{\left|\Gamma_{g}-\Gamma_{\mathrm{opt}}\right|^{2}}{\left|1+\Gamma_{\mathrm{opt}}\right|^{2}\left(1-\left|\Gamma_{g}\right|^{2}\right)}
$$

and determine unknown receiver noise parameters: the minimum noise factor $F_{\min }$, the equivalent noise resistance $R_{n}$, and the optimum signal-source reflection coefficient for minimum noise $\Gamma_{\text {opt }}$, where $Z_{0}$ is the characteristic impedance of the system, usually $50 \Omega$, and $\Gamma_{g}$ is the signal generator reflection coefficient seen by the device that, in the case of the receiver, is equal to $\Gamma_{i \text { Net }, i}^{c}$.

Step 4: Receiver Gain Extraction: We next consider three different approaches for determination of $G_{\text {rec }}$. For ease of addressing these approaches in this paper, we classify them by their methodologies, which include the simplified Cold method and two more accurate methods that account for changing reflection coefficient of the noise source as it toggles from ON to OFF.

Step 4a: Simplified Cold method [3], [5], [8]: As mentioned above, to use (7) for evaluation of $G_{\text {rec }}$, one needs to determine $P_{\text {rec,cal }}^{c(h)}$. These, however, are unavailable since the noise parameters of the receiver are not known. In a simplified approach, it 
is assumed that $\Gamma_{\mathrm{ns}}^{c} \approx \Gamma_{\mathrm{ns}}^{h}$, which results in $P_{\text {rec,cal }}^{h} \approx P_{\text {rec,cal }}^{c}$ and $G_{A, i \mathrm{Net}}^{c} \approx G_{A, i \mathrm{Net}}^{h}$, thus enabling the derivation of $G_{\mathrm{rec}}$ by dividing the difference of $P_{\text {cal }}^{h}$ and $P_{\text {cal }}^{c}$ in (7) by $T_{\mathrm{in}}^{h}-T_{\mathrm{in}}^{c}$ with the help of (6) and (B.5) to obtain

$$
k B G_{\mathrm{rec}} \approx \frac{P_{\mathrm{cal}}^{h}-P_{\mathrm{cal}}^{c}}{T_{\mathrm{in}}^{h}-T_{\mathrm{in}}^{c}} \frac{1}{G_{A, i \mathrm{Net}}^{c} M^{c}} .
$$

$G_{\text {rec }}$, obtained with (11), is used in (5) and (9) to extract the receiver noise parameters. This step is also simplified by assuming that $T_{\mathrm{ns}}^{c} \approx T_{a, 0}^{c(h)}$, in which case, from (6) and (B.5)

$$
P_{\mathrm{in}, i}^{c} \approx k B T_{a, 0}^{c}
$$

and $P_{\mathrm{in}, i}^{c}$ is no longer a function of InputNet $S$-parameters, and thus, calculations of (9) and (11) do not require the availability of InputNet $S$-parameters. Since measurement of InputNet $S$-parameters is a time-consuming task, this simplification reduces the calibration time by requiring only measurements of tuner output reflection coefficients $\Gamma_{i \mathrm{Net}, i}^{c}$ rather than a full set of $S$-parameters.

Step 4b: Iterative Cold Method [50]: The authors of [50] introduced an iterative approach to estimating the receiver's $G_{\text {rec }}$ parameters when $\Gamma_{\mathrm{ns}}^{h} \neq \Gamma_{\mathrm{ns}}^{c}$. In this method, an initial guess regarding the receiver noise parameters leads to finding $P_{\text {rec,cal }}^{c(h)}$ and to evaluation of the receiver's $G_{\mathrm{rec}}$ from (7) by

$$
G_{\text {rec }}=\frac{P_{\text {cal }}^{h}-P_{\text {cal }}^{c}}{\left(P_{\text {in }}^{h}+\frac{T_{a, 0}^{h}}{T_{0}} P_{\text {rec,cal }}^{h}\right) M^{h}-\left(P_{\mathrm{in}}^{c}+\frac{T_{a, 0}^{c}}{T_{0}} P_{\text {rec, cal }}^{c}\right) M^{c}} .
$$

This $G_{\text {rec }}$ gives $G_{T, \text { rec }}^{c}$ through (5), which is then used in (9) and (10) to determine $F_{\mathrm{min}}^{\mathrm{rec}}, R_{n}^{\mathrm{rec}}$, and $\Gamma_{\mathrm{opt}}^{\mathrm{rec}}$ that lead to a new estimate of $G_{\text {rec }}$ from (13). After a few iterations, at least three, as suggested in [50], this algorithm converges to a value of $G_{\text {rec }}$ and a set of noise parameters.

Step 4c: Direct Cold Method [4]: A method of estimating $G_{\text {rec }}$ and simultaneously deriving the noise parameters by using a direct least squares extraction approach rather than employing the iterative approach is discussed next. In this method, unknown $G_{T, \text { rec }, i}^{c}$ in (9) is expressed in terms of $P_{\text {cal }}^{h}$ with help from (7) and (5) transforming (9) to

$$
F_{\mathrm{rec}, i}=1+\frac{M^{h}}{M_{i}^{c}} \frac{P_{\mathrm{cal}, i}^{c}\left(P_{\mathrm{in}}^{h}+\frac{T_{a, 0}^{h}}{T_{0}} P_{\mathrm{rec}, \mathrm{cal}}^{h}\right)}{k B T_{a, i}^{c} P_{\mathrm{cal}}^{h}}-\frac{P_{\mathrm{in}, i}^{c}}{k B T_{a, i}^{c}}
$$

where $1 \leq i \leq N$ and subscript " 0 " refers to measurements in Step 2. After some algebraic manipulations, (14) results in

$$
Y_{\mathrm{rec}, i}^{\prime} F_{\mathrm{rec}, i}-F_{\mathrm{rec}}^{h}=\underbrace{\frac{T_{\mathrm{in}}^{h}-T_{a, 0}^{h}}{T_{a, 0}^{h}}+Y_{\mathrm{rec}, i}^{\prime} \frac{T_{a, i}^{c}-T_{\mathrm{in}, i}^{c}}{T_{a, 0}^{h}}}_{E_{\mathrm{rec}, i}^{\prime}}
$$

where $F_{\text {rec }}^{h}$ is the noise factor of the receiver driven by the InputNet, while the noise source is ON, and for brevity, we define

$$
Y_{\mathrm{rec}, i}^{\prime} \equiv \frac{T_{a, i}^{c}}{T_{a, 0}^{h}} \frac{M_{i}^{c}}{M^{h}} \frac{P_{\mathrm{cal}}^{h}}{P_{\mathrm{cal}, i}^{c}} .
$$

The determination of the receiver noise parameters proceeds by substituting (10) in place of $F_{\mathrm{rec}, i}$ and $F_{\text {rec }}^{h}$ in (15), calculating $E_{\text {rec }}^{\prime}$ based on measured $P_{\text {cal }}^{c(h)}$. This results in a set of $N$ equations in which all quantities are known, except for the noise parameters, which are obtained using a least squares fit over the set of the equations [11], [46]. Having obtained the receiver noise parameters, $G_{\text {rec }}$ can be calculated by using (13) to calculate $G_{\mathrm{rec}, i}$ for every measurement

$$
G_{\mathrm{rec}, i}=\frac{P_{\mathrm{cal}}^{h}-P_{\mathrm{cal}, i}^{c}}{\left(P_{\mathrm{in}}^{h}+\frac{T_{a, 0}^{h}}{T_{0}} P_{\mathrm{rec}, \mathrm{cal}}^{h}\right) M^{h}-\left(P_{\mathrm{in}, i}^{c}+\frac{T_{a, i}^{c}}{T_{0}} P_{\mathrm{rec}, \mathrm{cal}, i}^{c}\right) M_{i}^{c}}
$$

and then averaging $G_{\mathrm{rec}, i}$ to obtain $G_{\mathrm{rec}}$. Another option to obtaining $G_{\text {rec }}$ is by performing a least squares fit of (9) to measured $P_{\mathrm{cal}, i}^{c}$ with newly found receiver noise parameters employed to determine $F_{\text {rec }, i}$. We use the latter approach in the simulations and measurements presented in Section III.

Step 5: DUT Measurement: Once the receiver noise parameters and $G_{\text {rec }}$ are known, the DUT is inserted and the tuner is again manipulated to present a set of $N \Gamma_{i \text { Net }, i}^{c}$. The receiver reports $N$ noise power measurements denoted by $P_{\text {meas }, i}^{c}, 1 \leq$ $i \leq N$, which are functions of ambient temperatures $T_{a, i}^{c}$ that are also measured during this test. The measured $P_{\text {meas }, i}^{c}$ depend on noise powers of the InputNet, DUT, and receiver, amplified by appropriate gains, as shown in Appendix II-B. Based on the measurements of $P_{\text {meas }, i}^{c}$, the effective noise temperatures of the system at the DUT input at $T_{a, i}^{c}$ are calculated by

$$
T_{\text {meas }, i}^{c}=\frac{P_{\text {meas }, i}^{c}}{k B M_{\mathrm{DUT}, i}^{c} G_{\mathrm{rec}} G_{A, i}^{\mathrm{DUT}, c}}
$$

whereas the effective input-referred noise temperatures of the receiver alone at the DUT plane, $T_{\text {rec,meas }, i}^{c}$, at $T_{a, i}^{c}$ are calculated by

$$
T_{\text {rec }, \text { meas }, i}^{c}=\frac{T_{a, i}^{c}}{T_{0}} \frac{P_{\text {rec,meas }, i}^{c}}{k B G_{A, i}^{\text {DUT, }}} .
$$

During the DUT measurement, $P_{\text {rec,meas }, i}^{c}$ in (19) is calculated with receiver noise parameters from Step 4 and $M_{\mathrm{DUT}, i}^{c}$ is the receiver input mismatch factor when driven by the DUT. From (2), (18), and (19), the noise factors of the DUT for each $\Gamma_{\text {out }, i}$ are

$$
F_{\mathrm{DUT}, i}=1+\frac{T_{\mathrm{meas}, i}^{c}}{T_{a, i}^{c}}-\frac{T_{\mathrm{rec}, \mathrm{meas}, i}^{c}}{T_{a, i}^{c}}-\frac{T_{\mathrm{in}, i}^{c}}{T_{a, i}^{c}}
$$

where the last term removes the noise contribution of InputNet and the noise source.

In (20), all parameters are known, which leads to the determination of $F_{\mathrm{DUT}, i}$ from which the DUT's noise parameters are obtained by fitting (10) to $F_{\mathrm{DUT}, i}$ and extracting the DUT's noise parameters. In contrast with the receiver calibration, there is no need to determine the gain of the DUT, as it is known from its $S$-parameters.

Again, it is common to simplify (20) by assuming that $T_{a, i}^{c}$ are constant and equal to $T_{\mathrm{ns}}^{c}$. In this case, $T_{\mathrm{in}}^{c}$ in (20) no longer depends on full $S$-parameters of InputNet, thus simplifying the 
calibration procedure. This simplification is usually made when Step $4 a$ is used during receiver calibration.

\section{Modified Y-Factor Method}

The $Y$-factor method used in noise figure analyzers [36], [37] does not allow for DUT noise parameter extractions. Therefore, the modified $Y$-factor method has been devised that permits the creation of different signal-source impedances at the DUT input by either inserting a tuner as in [8]-[10] or a few known loads [11] to alter the source impedance. By measuring the resultant noise factors at each of the signal-source impedances, the DUT's noise parameters are de-embedded. The general system configuration shown in Fig. 2 still applies. Although in [11] the tuner is replaced by a passive network with variable loads that are attached in a sequence to generate the desired variable signal-source impedance, in general the behavior of the two systems is the same.

Step 1: S-Parameter Measurement: Similar to Step 1 in Section II-B, $S$-parameters and reflection coefficients are measured.

Step 2: Receiver Measurement: As in the case of the Cold methods, first the receiver is calibrated. To do this, the DUT is replaced by a "THRU" in Fig. 2 and $N(N>4)$ different impedances are presented to the receiver input by the tuner. At each such impedance, the noise source toggles from ON to OFF and different noise power levels $P_{\mathrm{cal}, i}^{c(h)}$ are reported by the noise figure analyzer. As shown in (4), $P_{\mathrm{cal}, i}^{c(h)}$ relate to receiver noise power and gain, repeated here for convenience as follows:

$$
\left\{\begin{array}{l}
P_{\mathrm{cal}, i}^{c}=\left(P_{\mathrm{in}, i}^{c}+\frac{T_{a, i}^{c}}{T_{0}} P_{\mathrm{rec}, \mathrm{cal}, i}^{c}\right) G_{T, \mathrm{rec}}^{c} \\
P_{\mathrm{cal}, i}^{h}=\left(P_{\mathrm{in}, i}^{h}+\frac{T_{a, i}^{h}}{T_{0}} P_{\mathrm{rec}, \mathrm{cal}, i}^{h}\right) G_{T, \mathrm{rec}}^{h} .
\end{array}\right.
$$

where $1 \leq i \leq N$. In (21), $P_{\text {rec,cal, } i}^{c(h)}$ and $G_{T \text {,rec }}^{c(h)}$ are unknown and ambient temperatures $T_{a, i}^{c(h)}$ are measured for each tuner setting. From these measurements, $N Y$-factors are obtained from

$$
Y_{\mathrm{rec}, i}=\frac{P_{\mathrm{cal}, i}^{h}}{P_{\mathrm{cal}, i}^{c}}
$$

which are dependent on receiver effective input noise temperatures and noise temperatures of the InputNet, as shown in Appendix II-C. The receiver noise factors $F_{\mathrm{rec}, i}^{c}$ expressed in terms of measured noise powers $P_{\mathrm{cal}, i}^{c}$ and calculated powers $P_{\mathrm{in}, i}^{c}$ by (6) were shown in (9) and repeated here as follows:

$$
F_{\mathrm{rec}, i}^{c}=1+\frac{P_{\mathrm{cal}, i}^{c}}{k B G_{T, \mathrm{rec}, i}^{c} T_{a, i}^{c}}-\frac{P_{\mathrm{in}, i}^{c}}{k B T_{a, i}^{c}} .
$$

In (23), however, there is an unknown $G_{\mathrm{rec}}$ that constitutes a part of $G_{T, \text { rec }, i}^{c}$ according to (5). We can derive an expression for $G_{\text {rec }}$ using the information obtained through Hot measurements from (5) as

$$
G_{\mathrm{rec}}=\frac{G_{T, \mathrm{rec}, i}^{h}}{M_{i}^{h}}=\frac{P_{\mathrm{cal}, i}^{h}}{P_{\mathrm{in}, i}^{h}+\frac{T_{a, i}^{h}}{T_{0}} P_{\mathrm{rec}, \mathrm{cal}, i}^{h}} \frac{1}{M_{i}^{h}}
$$

where the only unknowns are $P_{\text {rec,cal }, i}^{h}$. By expressing $G_{T, \text { rec }, i}^{c}$ through (24) and (5), substituting the result into (23) and by using (22) and (6), we obtain

$$
\begin{aligned}
Y_{\mathrm{rec}, i}^{\prime} F_{\mathrm{rec}, i}^{c}- & F_{\mathrm{rec}, i}^{h} \\
= & \underbrace{Y_{\mathrm{rec}, i}^{\prime}\left(\frac{T_{a, i}^{c}-T_{\mathrm{in}, i}^{c}}{T_{a, i}^{h}}\right)+\left(\frac{T_{\mathrm{in}, i}^{h}-T_{a, i}^{h}}{T_{a, i}^{h}}\right)}_{E_{\mathrm{rec}, i}^{\prime}}
\end{aligned}
$$

where

$$
Y_{\mathrm{rec}, i}^{\prime} \equiv \frac{T_{a, i}^{c}}{T_{a, i}^{h}} \frac{M_{i}^{c}}{M_{i}^{h}} Y_{\mathrm{rec}, i}
$$

and

$$
F_{\mathrm{rec}, i}^{h}=1+\frac{P_{\mathrm{rec}, \mathrm{cal}, i}^{h}}{k B T_{0}} .
$$

Equation (25) is very similar (15) and is the same as obtained in [11], however, intentionally obtained by using $G_{\text {rec }}$ to highlight the point that the modified $Y$-factor method implicitly calculates the receiver gain at each measurement point, and thus, $M_{i}^{c(h)}$ appear in (25) and (26). The similarity of (25) to (15) highlights an important observation that the tuner-based Cold methods are a subset of the modified $Y$-factor method.

Step 3: Receiver Noise Parameter Determination: A set of $N$ values of $E_{\text {rec }, i}^{\prime}$ are calculated with (22), (25), and (26) based on measured noise powers $P_{\mathrm{cal}, i}^{c(h)}$. The noise factor expression (10) is then substituted into (25) and the receiver noise parameter extraction proceeds by using one of a number of extraction algorithms available [11], [46]-[49] to determine the receiver's noise parameters that fit the set of $E_{\mathrm{rec}, i}^{\prime}$. This process is the same as in Step $4 c$ in Section II-B and can be used to find $G_{\text {rec }}$ if desired.

Step 4: DUT Measurement: When the receiver noise parameters are known, the DUT is inserted and the tuner is again manipulated to present a set of $N$ impedances to the DUT input. For each impedance, system $Y$-factors are calculated from measured noise powers $P_{\text {meas }, i}^{c(h)}$ by

$$
Y_{\mathrm{sys}, i} \equiv \frac{P_{\text {meas }, i}^{h}}{P_{\text {meas }, i}^{c}}
$$

The measured noise powers used to define the $Y$-factors in (28) consist of known quantities as shown in Appendix II-D and the unknown $P_{\mathrm{DUT}, i}^{c(h)}$ that are related through DUT noise parameters. Rearranging the expanded version of (28) shown in (B.10), one obtains

$$
Y_{\mathrm{sys}, i}^{\prime \prime} F_{i}^{c}-F_{i}^{h}=E_{\mathrm{sys}, i}^{\prime}
$$

where

$$
Y_{\mathrm{sys}, i}^{\prime \prime} \equiv \underbrace{Y_{\mathrm{sys}, i} \frac{M_{\mathrm{DUT}, i}^{c}}{M_{\mathrm{DUT}, i}^{h}}}_{Y_{\mathrm{sys}, i}^{\prime}} \frac{G_{A, i}^{\mathrm{DUT}, c}}{G_{A, i}^{\mathrm{DUT}, h}} .
$$

The effective ENR of the system is then

$$
\begin{aligned}
E_{\mathrm{sys}, i}^{\prime} \equiv \frac{T_{\mathrm{in}}^{h}-T_{a, i}}{T_{a, i}} & -Y_{\mathrm{sys}, i}^{\prime \prime}\left(\frac{T_{\mathrm{in}}^{c}-T_{a, i}}{T_{a, i}}\right) \\
& -\frac{Y_{\mathrm{sys}, i}^{\prime} F_{\mathrm{rec}, i}^{c}-F_{\mathrm{rec}, i}^{h}+1-Y_{\mathrm{sys}, i}^{\prime}}{G_{A, i}^{\mathrm{DUT}, h}} .
\end{aligned}
$$


TABLE I

SIMULATION PARAMETERS

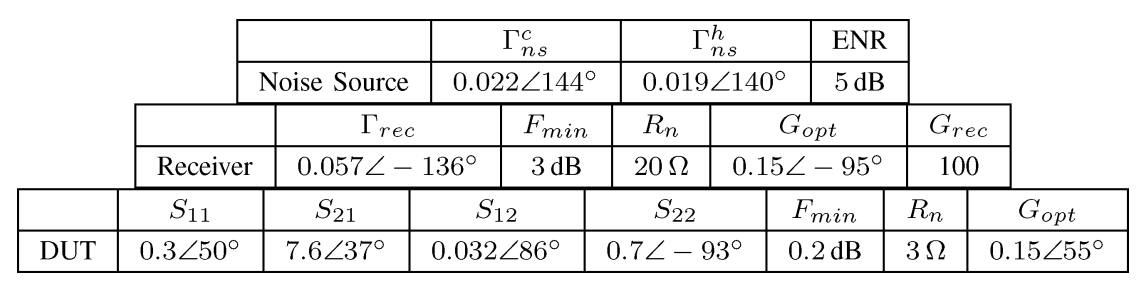

$N$ sets of $Y_{\mathrm{sys}, i}^{\prime \prime}$ and $E_{\mathrm{sys}, i}^{\prime}$ are calculated from (30) and (31) and are based on measured $P_{\text {meas }, i}^{c(h)}$ through (28). Having found $Y_{\mathrm{sys}, i}^{\prime \prime}$ and $E_{\mathrm{sys}, i}^{\prime}$ and by substituting the general expression of the noise factor shown in (10) into (29), the noise parameters of the DUT are found by fitting the resultant $N$ expressions to measured data [11].

\section{COMPARISON OF NoISE PARAMETER MEASUREMENTS}

\section{A. Monte Carlo Based Comparison of the Extraction Techniques}

To verify the expressions discussed in Section II and to evaluate the performance of the Cold methods and the modified $Y$-factor method, a Monte Carlo analysis was performed. In this analysis, Agilent's Advanced Design System (ADS) software was used to generate the data similar to what would be measured in a real measurement environment. The generated data were then fed to data processing software written in MATLAB. This software added measurement uncertainties to the data, as discussed in Section III-A.1, and extracted the noise parameters.

In ADS, the "tuner" was implemented with two ideal transmission lines with variable phases separated by a variable shunt capacitor. A loss was added with a small resistor $(2 \Omega)$ in series with the transmission lines. The "tuner" $S$-parameters at all settings of transmission line phases and variable capacitor values were recorded. The modification of the transmission line phases and the value of the shunt capacitor allowed generation of a large number of impedances fully covering the Smith chart. The corresponding power delivered to the output port of the receiver for each impedance generated by the "tuner" was recorded for both "calibration" step and the "measurement" step. The noise source was implemented with a port that had a nonzero reflection coefficient and its operating temperature was specified based on both Hot and Cold states, depending on which one was being measured.

The ambient temperature drift over time was simulated with a train of random numbers varying between $-1.5^{\circ} \mathrm{C}$ and $1.5^{\circ} \mathrm{C}$ filtered with a Chebyshev fifth-order low-pass filter having its cutoff frequency, assigned arbitrarily, to 100/3 times slower than the rate at which the "measurements" are performed. This filtered signal was applied to ambient temperature with an assumption that Hot and Cold ambient temperature readings for the same tuner setting are equal. In these simulations, it was assumed that the noise power of all devices, active and passive, linearly scales with ambient temperature, as discussed in Section II. In all simulations, $T_{\mathrm{ns}}^{c}$ was $5 \mathrm{~K}$ higher than $T_{a}^{c}$ in accordance with our experience of using N4000A noise sources.
Performance of tuner-based noise parameter extraction methods is dependent on the constellation of impedances presented by the source tuner to the DUT [2], [51]-[54]. To compare performance of the different noise parameter extraction algorithms, two nine-point impedance constellations are selected by both the method in [53] and randomly. The random constellation is selected to always possess an impedance point near the center of the Smith chart as it produces the best estimate of $G_{\text {rec }}$ in Cold methods [2], [50], [51]. The same constellations were given to all four noise parameter extraction methods. The results of noise parameter extractions presented in Section III-A.2, the receiver "calibrations" and DUT "measurements" were performed 1000 times. Having obtained data for this large sample of extracted noise parameters allows us to use statistical approaches, a common practice for this type of work [53], [55], [56], to determine whether there are underlying inaccuracies in the measurement methods themselves.

The noise parameter extractions require a numerical analysis of measured data as discussed in [46], [47] and [57]-[59]. A few numerical noise parameter extraction algorithms employed by tuner-based measurement systems have been compared in [57] for the Cold method where it was shown that the extraction technique proposed in [58] and [59] is less sensitive to the constellation of the signal-source impedances presented by the source tuner. However, the least squares fit techniques proposed in [46] and [47] can be improved by applying a scaling factor. When the scaling factor is applied, the accuracy of [46] and [47] was found to be similar to [58] and [59]. In this paper, both the Cold method and modified $Y$-factor method are analyzed. An equivalent extraction least squares fit technique to [46], modified as in [57], is applied to the modified $Y$-factor method, as discussed in [11].

In all simulations that are based on the iterative Cold method in Step $4 b$ in Section II-B, we used ten iteration steps, which is larger than three iterations reported in [50] as being sufficient for noise parameter extraction.

The simulation parameters are summarized in Table I. These parameters are closely based on the real measurement system used in our laboratory at $1.4 \mathrm{GHz}$ and on a typical LNA (labeled "DUT" in Table I) that may be used with the SKA radio telescope. The performance of the noise parameter extraction algorithms was verified by comparing their outputs to the simulated parameters given to ADS. These two sets agreed perfectly in the absence of uncertainties discussed below.

1) Measurement Uncertainties: To provide realistic "measurement" conditions, noise was added to all measured quantities required to extract the noise parameters. The following list describes simulated uncertainties: 
TABLE II

Measurement Uncertainties Added to Evaluate Noise Parameter Extraction Methods Based ON THE INSTRUMENT SPECIFICATIONS NOTED IN THE REFERENCE COLUMN

\begin{tabular}{|l||c|c|c|c|}
\hline Measured parameter & Uncertainty & Noise model & Note & References \\
\hline \hline \multirow{2}{*}{ S-parameters } & $\begin{array}{c}\text { mean }=-65 \mathrm{~dB} \\
\mathrm{max}=-60 \mathrm{~dB}\end{array}$ & Rayleigh & High power, Notes 1,2 & [60] \\
\cline { 2 - 4 } & $\begin{array}{c}\mathrm{mean}=-55 \mathrm{~dB} \\
\mathrm{max}=-50 \mathrm{~dB}\end{array}$ & Rayleigh & Low power, Notes 1,2,3 & \\
\hline Tuner repeatability & $\begin{array}{c}\mathrm{mean}=-50 \mathrm{~dB} \\
\mathrm{max}=-44 \mathrm{~dB}\end{array}$ & Rayleigh & Notes 4,5 & {$[61]-[63]$} \\
\hline$T_{c}$ measurement accuracy & $\begin{array}{c}\text { mean }=0.25^{\circ} \mathrm{C} \\
\text { st. dev. }=0.33^{\circ} \mathrm{C}\end{array}$ & Gaussian & Note 6 & {$[64]$} \\
\hline Receiver accuracy & $0.017 \mathrm{~dB}$ & Gaussian & Note 7 & {$[65]$} \\
\hline Noise source ENR & $0.134 \mathrm{~dB}$ & Constant offset & Note 8 & {$[64]$} \\
\hline
\end{tabular}

Note 1: Since the uncertainties in the real and imaginary parts of S-parameter measurements are correlated by an unknown amount [66], the uncorrelated real and imaginary parts of S-parameters were selected as they produce larger errors in the extracted noise parameters

Note 2: Values are based on the authors calibration experience with TRL kits using 128 averages and [60]

Note 3: When measuring the receiver input reflection coefficient and the DUT S-parameters, the VNA output power needs to be backed off in order to avoid compression of the two devices. Depending on linearity specifications of the DUT, power levels of less than $-40 \mathrm{dBm}$ are required

Note 4: It was assumed that phase errors and magnitude errors are not correlated

Note 5: Some automatic tuner vendors allow users to perform noise measurements at tuner locations that have not been calibrated but rather the supplied software estimates the actual reflection coefficient values based on measured surrounding points. Based on our measurements the estimated points worsen the tuner repeatability to a mean value of $-35 \mathrm{~dB}$ with the maximum difference reaching $-22 \mathrm{~dB}$

Note 6: To assess the effect of errors in measurements of $T_{a}$ and $T_{n s}^{c}$, a constant offset representing a reasonable accuracy of temperature measurements of $0.25 \mathrm{~K}$ was introduced for both temperature "readings" used by the Matlab program to extract the noise parameters

Note 7: When the noise figure analyzer bandwidth is $4 \mathrm{MHz}$

Note 8: Average ENR uncertainty for an Agilent noise source N4000A in the authors' possession

S-Parameters and Reflection Coefficients: These uncertainties simulate errors in the VNA calibration and have been shown to affect the accuracy of the noise parameter measurements [67]. Based on our experience with a calibrated VNA and [60], the uncertainties in the $S$-parameters were set as shown in Table II and were applied to all "measured" $S$-parameters and reflection coefficients ${ }^{5}$ using a Rayleigh distribution.

Tuner Repeatability: This measurement error is defined as the magnitude change in the $S$-parameters of the tuner after it had been manipulated and then returned to the original setting. This repeatability error, usually reported in decibels, as shown in Table II, was included in the simulations of the noise parameter measurement system based on the tuner specifications in [61]-[63] and was simulated with Rayleigh distribution.

Temperature Measurement: Gaussian noise was added to all temperature "readings" to simulate uncertainty of the temperature sensors. It was assumed that as the ambient temperature drifts $^{6,7}$ so does the Cold noise temperature of the noise source.

\footnotetext{
${ }^{5}$ The other source of error is the difference between the VNA measurement bandwidth and the receiver measurement bandwidth. This difference creates an unknown error in the phase and magnitude of the measured $S$-parameters. In these simulations, we are assuming that all devices measured do not exhibit very rapid change in their $S$-parameters with frequency, and therefore, this source of error is ignored.

${ }^{6}$ A DUT consuming a large amount of power and warming up adjacent measurement system components can create errors in the measurements. This can be remedied by modifying the ambient temperature used in calculations of each system component's noise powers by the amount of temperature rise. This modification is not shown in this paper and is not analyzed any further since most DUTs are relatively low-power devices and do not cause temperature rise in the adjacent components

${ }^{7}$ The temperature gradient resulting from the temperature difference between $T_{c}^{c}$ and $T_{\text {ns }}^{c}$ has been ignored in these simulations and is a subject of future study. This gradient adds an additional source of uncertainty and sometimes is dealt with by controlling the room temperature [11] and by reducing losses of the InputNet. It was also assumed that for a given tuner setting, Cold and Hot measurements happen relatively fast such that $T_{a}^{c}=T_{a}^{h}$.
}

Receiver Measurement Accuracy: Random Gaussian noise, based on a noise figure analyzer specifications [65], was added to the noise power delivered to the load to simulate errors introduced by the receiver during noise power measurement.

ENR Uncertainty: The new noise sources, such as Agilent's N4000A, report the uncertainty of the ENR values [64]. The uncertainty can be downloaded and used in the noise parameter extraction methods to estimate the accuracy of the final result. The $3 \sigma$ uncertainty averaged over all specified frequencies of four the noise sources in our possession is $0.134 \mathrm{~dB}$ with the maximum uncertainty of $0.174 \mathrm{~dB}$ and minimum of $0.104 \mathrm{~dB}$. Since the ENR values and the corresponding uncertainties are only specified at cardinal frequencies, there is an additional uncertainty when the ENR value is extrapolated to other frequencies. We assume that this is incorporated into the specified ENR uncertainty. Since a given noise source has an ENR that can only change over periods of time that are much longer than a typical measurement duration, we show the effect of ENR uncertainty on noise parameter extraction results for the case when the actual ENR is constant and is higher than specified by $0.134 \mathrm{~dB}$.

2) Discussions: Noise parameter extraction simulations were performed with "measurements" contaminated with noise that simulates measurement uncertainties in Table II. The simulation results are summarized in Figs. 3 and 4. The two figures show systematic errors and the standard deviations in the measured noise parameters of the receiver and DUT. There is one vertical bar for each extracted noise parameter for each extraction method. In these figures, the bottom-to-top patterning of the vertical bars is in sequence with the legend that identifies five "measured" quantities from Table II. The vertical numbers in these vertical bars indicate systematic offsets in Fig. 3 and standard deviations in Fig. 4 that are simulated when only the "measured" quantity of interested is uncertain. For example, 


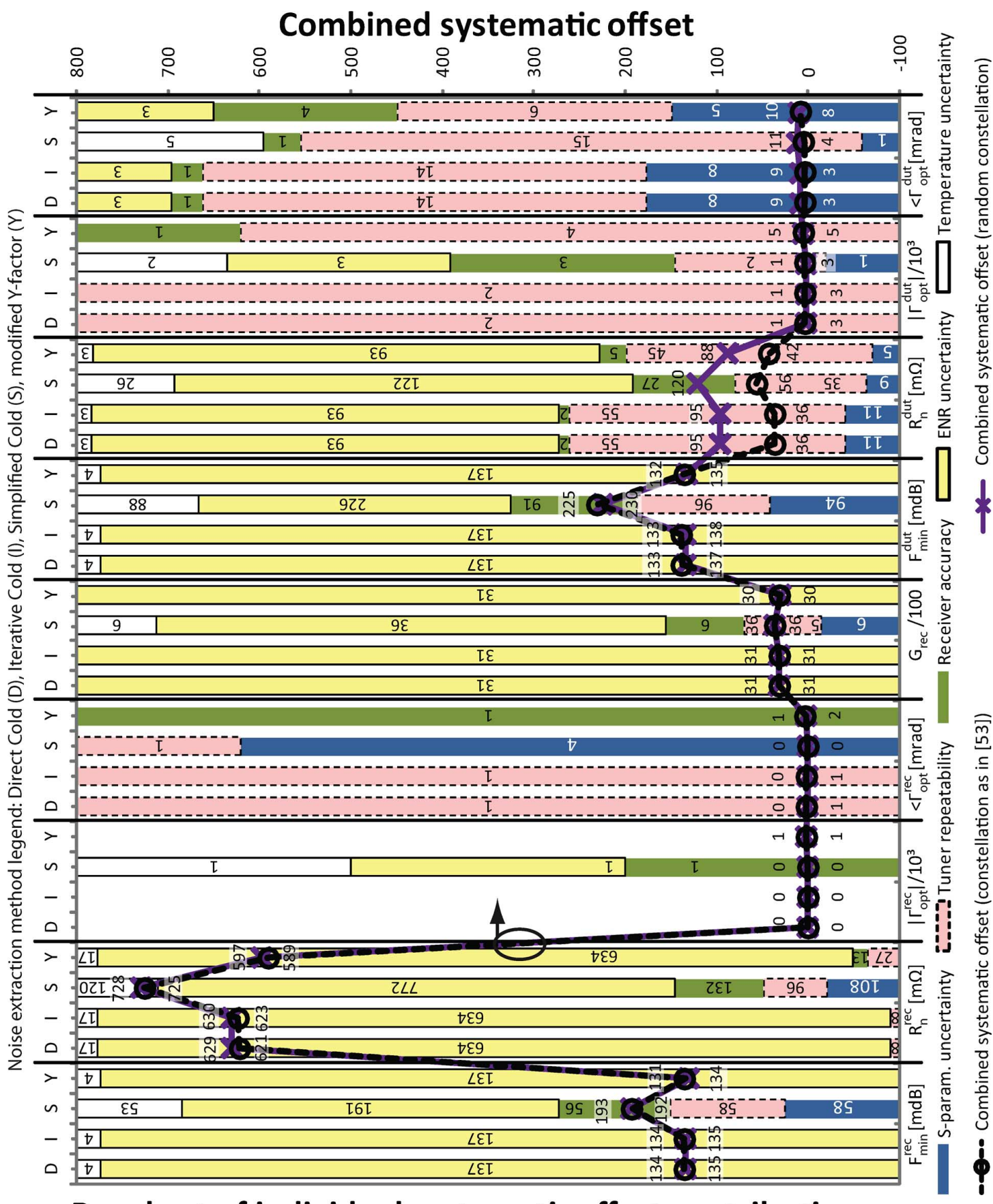

Bar chart of individual systematic offset contributions

Fig. 3. Systematic offsets in the noise parameter "measurements" of the receiver and the DUT, as well as the systematic error in $G_{\text {rec }}$ when impedance constellation in [53] is used. Values reported are the systematic errors obtained after 1000 system "calibrations" and DUT "measurements" by subtracting the true values in Table I from "measured" values. The bar chart part of this figure shows systematic offsets when only the individual uncertainty from Table II was introduced in the simulations. Combined systematic offset shows the systematic offset when all measurement uncertainties are included in the simulations. For comparison, combined systematic offsets obtained with randomly selected impedance constellations is also shown. The combined systematic-offset units relate to the quantities in question and are identified along the bottom $x$-axis. Systematic offsets that are less then $1 \%$ of the largest systematic offset for each "measured" quantity are not shown to improve readability. When the two combined systematic offsets are nearly on top of each other, the labels related to the random constellations are shown above the data symbols and the other labels are below the data symbols. Noise parameters of the receiver and the DUT are identified by appropriate superscripts.

from Figs. 3 and 4, the systematic offset and the $1 \sigma$ standard deviation in the $F_{\min }$ of the receiver, $F_{\min }^{\text {rec }}$, when measured with the simplified Cold method would be $58 \mathrm{mdB}$ from Figs. 3 and $\pm 5 \mathrm{mdB}$ from Fig. 4 if only $S$-parameter measurement had uncertainty. Similarly, the systematic offset and the standard deviations in $F_{\mathrm{min}}^{\mathrm{rec}}$ extracted using the simplified Cold method would be $191 \pm 0 \mathrm{mdB}$ if only the ENR uncertainty was present.
Figs. 3 and 4 also show the combined systematic offset and the combined standard deviation when all uncertainties are present. These are shown with the horizontal numbers and can be also read off the right-hand-side axis. One of the combined sets of data is obtained for the case when the impedance constellation is based on [53] and the other set is for the case when the impedance constellation is randomly selected. The 


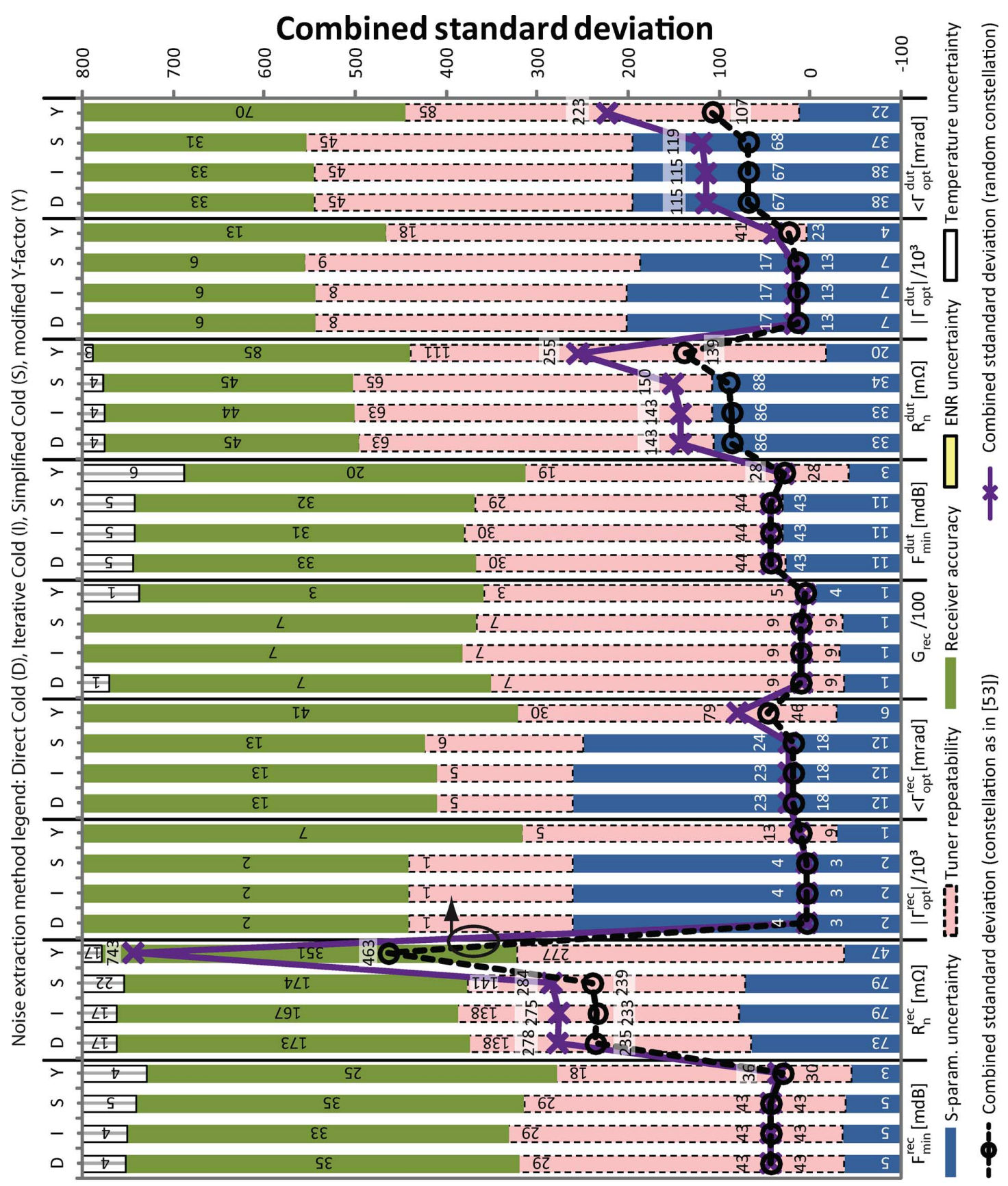

\section{Bar chart of individual standard deviations}

Fig. 4. $1 \sigma$ standard deviations in the noise parameter "measurements" of the receiver and the DUT, as well as the systematic error in $G_{\text {rec }}$ when impedance constellation in [53] is used. Values reported are the standard deviations obtained after 1000 system "calibrations" and DUT "measurements" by subtracting the true values in Table I from "measured" values. The bar chart part of this figure shows standard deviations when only the individual uncertainty from Table II was introduced in the simulations. Combined standard deviation shows the standard deviation when all measurement uncertainties are included in the simulations. For comparison, combined standard deviation obtained with randomly selected impedance constellations is also shown. The combined standard-deviation units relate to the quantities in question and are identified along the bottom $x$-axis. Standard deviations that are less then $1 \%$ of the largest standard deviation for each "measured" quantity are not shown to improve readability. When the two combined standard deviations are nearly on top of each other, the labels related to the random constellations are shown above the data symbols and the other labels are below the data symbols. Noise parameters of the receiver and the DUT are identified by appropriate superscripts.

combined systematic offsets and $1 \sigma$ standard deviations in the "measurement" of, for example, $F_{\min }$ of the DUT, $F_{\min }^{\mathrm{DUT}}$, using the simplified Cold method with the two impedance constellation approaches can be read from Figs. 3 and 4 as $230 \pm 43 \mathrm{mdB}$ and $225 \pm 44 \mathrm{mdB}$, respectively.

Figs. 3 and 4 demonstrate that performance of the two constellation selection methods are similar. However, constel- lation selection based on [53] shows smaller standard deviation, especially for extraction of $R_{n}$ (see Fig. 4). The systematic errors are slightly better with the randomly selected constellation for $R_{n}$ measurements as seen from Fig. 3. Based on the simulations carried out in this study, it appears that constellation selection according to [53] overall yields more accurate measurements. 


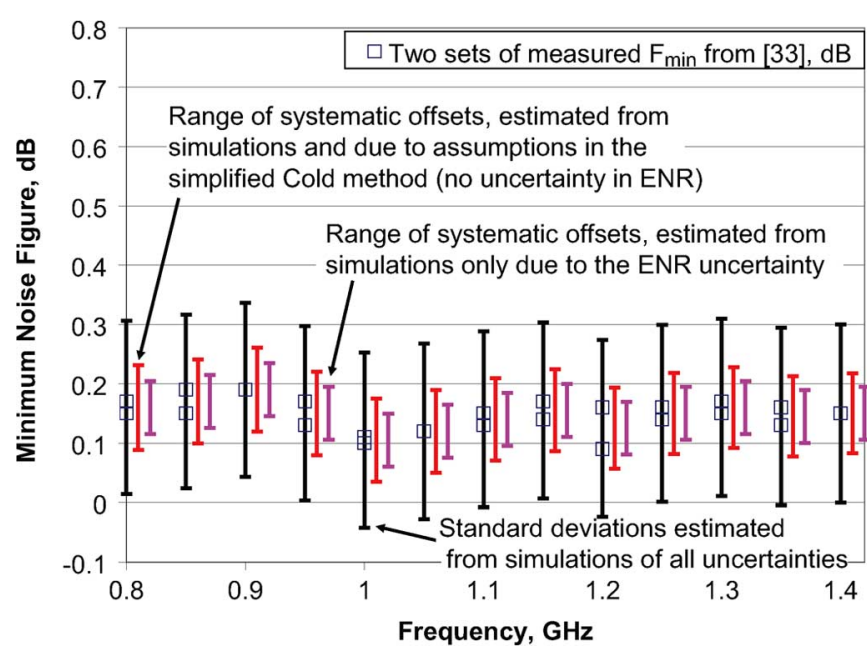

Fig. 5. Measured $F_{\min }$ from [33] with measurement uncertainty estimated with analysis in this paper denoted by error bars. $1 \sigma$ ENR uncertainty was used. The systematic offsets are result of the assumptions $\left(\Gamma_{\mathrm{ns}}^{c} \approx \Gamma_{\mathrm{ns}}^{h}\right.$ and $\left.T_{\mathrm{ns}}^{c} \approx T_{a, o}^{c(h)}\right)$ made in the simplified Cold method, as discussed in Section II-B (Step $4 a$ ) and the other systematic offsets are due to the ENR uncertainty.

The simulations also show that the simplified Cold method is the least reliable for extraction of $F_{\min }$. The modified $Y$-factor method and the direct Cold and the iterative Cold methods have similar systematic offsets when extracting $F_{\min }$, but the modified $Y$-factor method has slightly smaller standard deviation in the measurement. The direct Cold method and the iterative Cold method perform similarly, and they are as good or better than the modified $Y$-factor method in all aspects other than the extraction of $F_{\min }$.

Assumptions that ambient temperature is constant throughout the receiver "calibration" and DUT "measurement" and that the noise source is at ambient temperature produce systematic offsets in the extracted $F_{\min }$ and $R_{n}$. These assumptions, made with the simplified Cold method, result in a systematic offset of $\sim 0.09 \mathrm{~dB}$ in the $F_{\min }^{\mathrm{DUT}}$ "measurement." This error depends on losses in the InputNet. Figs. 3 and 4 illustrate that extraction of $\Gamma_{\mathrm{opt}}$ is not sensitive to the accuracy of temperature measurements.

Fig. 3 demonstrates that the error in the ENR propagates through the measurements and adds directly to the resultant $F_{\min }$, creating a systematic offset. The error in ENR also directly affects $R_{n}$ and $G_{\text {rec }}$. Therefore, all ambient temperature tuner-based noise parameter extraction methods have uncertainty that is no better than the uncertainty in the ENR of the noise source.

Figs. 3 and 4 show that errors in $F_{\min }$ extraction of a lownoise DUT and of a relatively high-noise receiver are very similar in absolute terms. This indicates that an adequate measurement system for a relatively high noise figure DUT may be very inadequate for a very low-noise DUT.

Based on the systematic error and standard deviation simulations, we can estimate these statistical quantities in the noise parameter measurements from [33] that were performed using the simplified Cold method. Figs. 5-7 show results from [33] with the range of systematic offsets and standard deviations added to illustrate the estimated measurement uncertainty obtained from the simulations described in this paper.

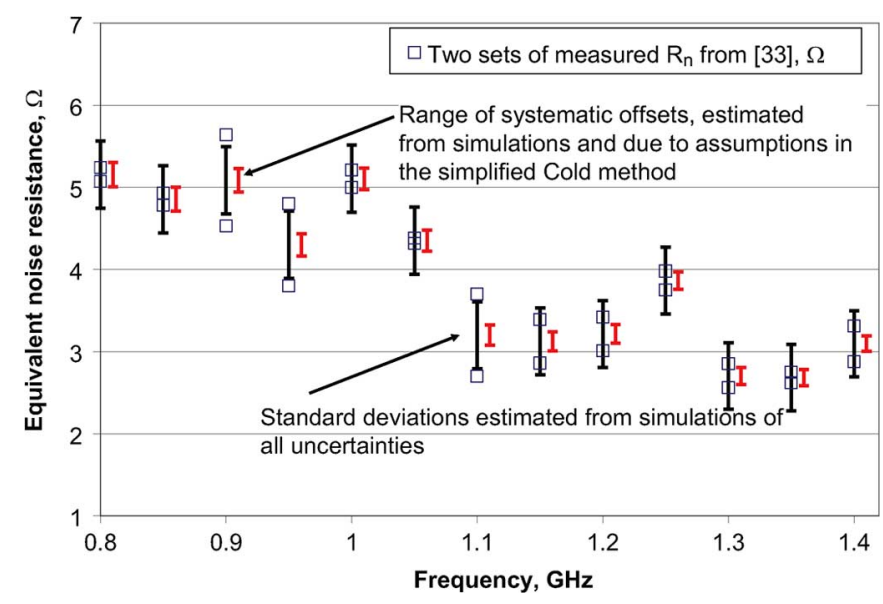

Fig. 6. Measured $R_{n}$ from [33] with measurement uncertainty estimated with analysis in this paper denoted by error bars. $1 \sigma$ ENR uncertainty was used. The systematic offset comes from the assumptions $\left(\Gamma_{\mathrm{ns}}^{c} \approx \Gamma_{\mathrm{ns}}^{h}\right.$ and $\left.T_{\mathrm{ns}}^{c} \approx T_{a, o}^{c(h)}\right)$ made in the simplified Cold method, as discussed in Section II-B (Step 4a) and the ENR uncertainty.

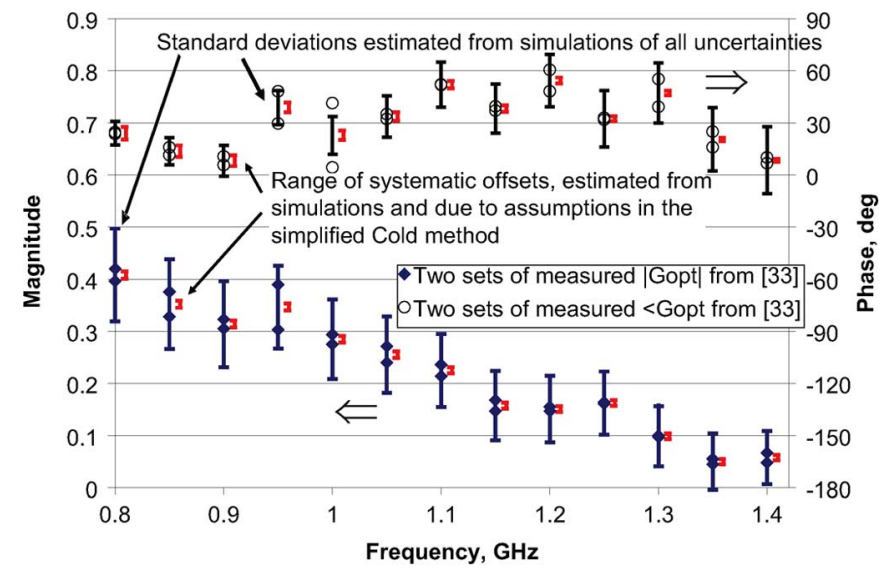

Fig. 7. Measured $\Gamma_{\mathrm{opt}}$ from [33] with measurement uncertainty estimated with analysis in this paper denoted by error bars. $1 \sigma$ ENR uncertainty was used. The systematic offset comes from the assumptions $\left(\Gamma_{\mathrm{ns}}^{c} \approx \Gamma_{\mathrm{ns}}^{h}\right.$ and $\left.T_{\mathrm{ns}}^{c} \approx T_{a, o}^{c(h)}\right)$ made in the simplified Cold method, as discussed in Section II-B (Step 4a) and the ENR uncertainty.

\section{B. Experimental Comparison of the Noise-Parameter Extraction Methods}

To verify the conclusions made based on the Monte Carlo analysis, a set of successive receiver calibrations employing the iterative Cold method were carried out. In these measurements, the receiver consisted of a noise figure analyzer with an RF switch connected by an RF cable. The switch allowed the in-situ measurement of both $\left[S^{i \text { Net }}\right]$ for all tuner settings and $\Gamma_{\text {rec. }}$. There were six tests performed, as summarized in Table III. Standard deviations of the measured data were calculated and are presented in Table IV. Analysis of these results suggest that one of the strongest influences on the data scatter is the number of averages employed. Test 3 shows a significant decrease in the standard deviations compared to all other methods. Measurements collected during Test 4 show a slight increase in their standard deviations indicating that perhaps temperature measurements do reduce data scatter. Standard deviations of measured quantities in Test 3 are smaller than those of Test 6 . If receiver accuracy were the dominant source of data scatter, results of Test 6 would have exhibited smaller standard deviations 

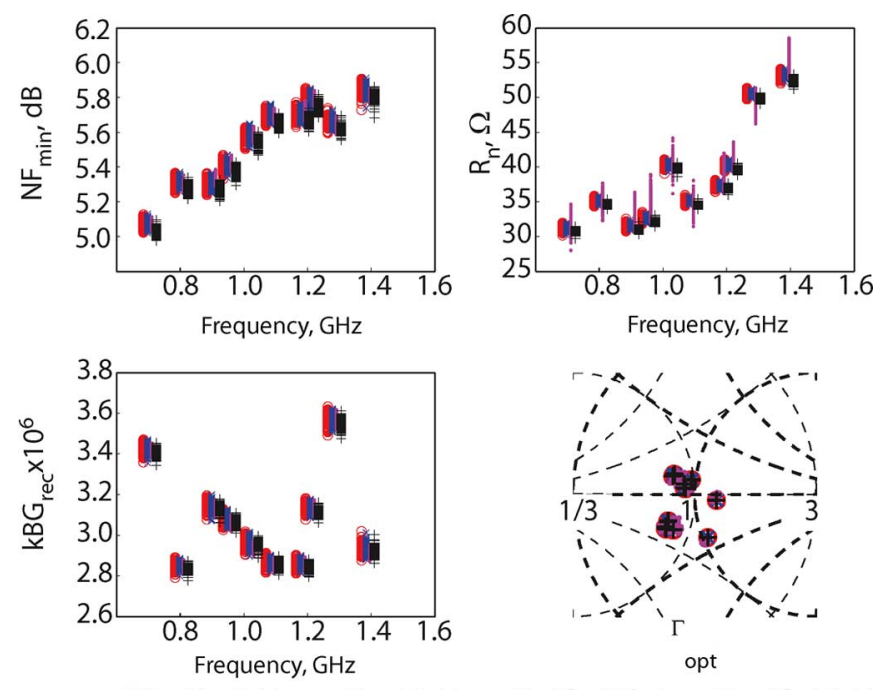

- Iterative Cold $\times$ Direct Cold - Modified Y-factor + Simplified Cold Fig. 8. Experimental comparison of noise-parameter extraction techniques. Noise parameters and $k B G_{\text {rec }}$ shown are for a receiver consisting of a noise figure analyzer, an RF switch, and an RF cable. The noise figure analyzer bandwidth was set to $1 \mathrm{MHz}$.

TABLE III

ReCEIVER Noise PARAMETER EXTRACTION TESTS. DURING Test $4, T_{a}$ WAs Set TO A Constant $24{ }^{\circ} \mathrm{C}$. ThE

NoIse Figure ANALYZER BANDWIDTH WAS SET TO $1 \mathrm{MHz}$

\begin{tabular}{|c||c|c|c|c|c|c|}
\hline Test & $\begin{array}{c}{\left[S^{i N e t}\right.} \\
\text { meas. }\end{array}$ & $\begin{array}{c}\Gamma_{r e c} \\
\text { meas. }\end{array}$ & $\begin{array}{c}\text { Receiver } \\
\text { ave. }\end{array}$ & $\begin{array}{c}T_{a} \\
\text { meas. }\end{array}$ & $\begin{array}{c}\text { No of } \\
\text { meas. }\end{array}$ & Constellation \\
\hline \hline 1 & Yes & Yes & 32 & Yes & 25 & {$[53]$} \\
\hline 2 & No & No & 32 & Yes & 25 & {$[53]$} \\
\hline 3 & No & No & 128 & Yes & 25 & {$[53]$} \\
\hline 4 & No & No & 32 & No & 25 & {$[53]$} \\
\hline 5 & No & No & 32 & Yes & 25 & $\begin{array}{c}\text { all 217 tuner } \\
\text { impedances }\end{array}$ \\
\hline 6 & No & No & 32 & Yes & 150 & {$[53]$} \\
\hline
\end{tabular}

since Test 6 was repeated six times more, whereas Test 3 had only four times longer receiver measurement averaging. Since results of Test 6 did not produce smaller standard deviations, the conclusion is that the tuner repeatability is a more significant source of measurement uncertainty than the receiver accuracy when the receiver employs 32 averages. Large standard deviations in measured data of Test 5 also indicate that tuner repeatability is a significant problem in the measurements. During each iteration of Test 1, [ $\left.S^{i \text { Net }}\right]$ for all tuner positions and $\Gamma_{\text {rec }}$ were measured. This, however, did not result in a decrease of the data scatter compared with other tests, indicating that the $S$-parameter measurement repeatability is sufficiently good and does not contribute significantly to measurement uncertainties. All these conclusions are consistent with results reported in Fig. 4.

To verify the conclusions from Section III-A.2 regarding relative performances of the noise-parameter extraction methods, additional measurements were conducted. In these measurements, 150 successive receiver calibrations were performed over a span of two days. During these measurements, impedance constellations based on [53] were used. The same data was given to all four noise parameter extraction methods and no in-situ $S$-parameter measurements were performed between the successive receiver calibrations. Fig. 8 shows the results of the measurements. Based on these measurements,
TABLE IV

Standard DeViations Obtained From MEASUREMENTS DuRing Tests 1-6. The NoISE Figure ANALYZER BANDWIDTH WAS SET to $1 \mathrm{MHz}$

\begin{tabular}{|c||c|c|c|c|c|}
\hline Test & $T_{\min }, \mathrm{K}$ & $R_{n}, \Omega$ & $\left|\Gamma_{\text {opt }}\right|$ & $\angle \Gamma_{\text {opt }}$ & $k B G_{\text {rec }}$ \\
\hline \hline 1 & 21 & 0.32 & 0.0018 & $1.8^{\circ}$ & $2.3 \mathrm{e}-8$ \\
\hline 2 & 20 & 0.31 & 0.0019 & $1.6^{\circ}$ & $1.7 \mathrm{e}-8$ \\
\hline 3 & 12 & 0.22 & 0.0017 & $1.0^{\circ}$ & $1.4 \mathrm{e}-8$ \\
\hline 4 & 20 & 0.31 & 0.0016 & $1.8^{\circ}$ & $2.0 \mathrm{e}-8$ \\
\hline 5 & 24 & 0.32 & 0.0031 & $1.3^{\circ}$ & $2.0 \mathrm{e}-8$ \\
\hline 6 & 18 & 0.31 & 0.0017 & $1.8^{\circ}$ & $1.8 \mathrm{e}-8$ \\
\hline
\end{tabular}

TABLE V

COMPARISON OF STANDARD DEVIATIONS FROM THE Four NOISE PARAMETER EXTRACTION METHODS NORMALIZED BY THE CORRESPONDING DATA ObTained With the Iterative COLD Method. The NoISE Figure ANALYZER BANDWIDTH WAS SET TO $1 \mathrm{MHz}$

\begin{tabular}{|c|c|c|c|}
\hline & Direct Cold & Modified Y-factor & Simplified Cold \\
\hline \hline$T_{\min }$ & 0.9 & 0.7 & 1.0 \\
\hline$R_{n}$ & 1.0 & 3.8 & 1.0 \\
\hline$\left|\Gamma_{\text {opt }}\right|$ & 1.0 & 4.4 & 1.0 \\
\hline$\angle \Gamma_{\text {opt }}$ & 1.0 & 5.0 & 1.0 \\
\hline$k B G_{r e c}$ & 0.9 & 0.7 & 1.0 \\
\hline
\end{tabular}

standard deviations of extracted noise parameters and $k B G_{\text {rec }}$ were calculated. These results, normalized by the standard deviations of data extracted with the iterative Cold method, are shown in Table V. The obtained standard deviations confirm that the modified $Y$-factor method produces the smallest standard deviations when extracting $T_{\min }$ and $k B G_{\text {rec }}$ while having the largest standard deviations when extracting $\Gamma_{\mathrm{opt}}$ and $R_{n}$. Inspection of Fig. 4 and Table $\mathrm{V}$ shows that results of the numerical simulations are well supported by the measurements. The experimental results also show offsets in mean values of the extracted parameters. While the true values are not known, the data demonstrate that the mean values of the noise parameters obtained with the simplified Cold method deviate the most from those obtained with the other three methods. This observation is consistent with findings in Section III-A.2.

\section{CONCLUSION}

This paper has presented a detailed description of the measurement procedures employing tuners to synthesize various signal-source impedances and allow for noise parameter extractions. The discussed methods are: the modified $Y$-factor method, direct Cold method, iterative Cold method, and simplified Cold method. The modified $Y$-factor method was shown to produce a slightly better estimate of $F_{\min }$ when all measurement uncertainties are included by having the lowest standard deviation in the measured data. However, the iterative and direct Cold methods are better at extraction of $R_{n}$ and $\Gamma_{\mathrm{opt}}$.

The paper discussed a method of assessing standard deviations and systematic offsets in these noise extraction methods. These standard deviations and the systematic offsets were applied to measurements of a very low-noise amplifier presented in [33] in order to illustrate the relative size of the uncertainties. The uncertainty in the noise source's ENR value is a large contributor to the systematic error in the noise parameter measurement, and therefore, higher quality cryogenic noise sources are often used to reduce this error. Assumption of a noise source 
Cold temperature being equal to the room temperature is shown to produce an error in the extracted noise parameters. The simplified Cold method suffers from this assumption and from the difference in the noise source Hot and Cold reflection coefficients. However, for a high noise figure, DUT resultant errors are less significant.

\section{APPENDIX I}

\section{NOTES ON NOTATIONS AND DEFINITIONS}

In this paper, superscripts $h$ and $c$ refer to measurements with a noise source turned ON ("Hot" state) and OFF ("Cold" state), respectively. Derivations of parameters for Cold and Hot states of the noise source are combined and identified by superscript $c(h)$. The following are symbols and subscripts used (Fig. 2 is used for graphical explanation of some of the parameters).

- Subscript $i$ : Indicates that the measurements are performed for $1 \leq i \leq N$ tuner positions. A special case of $i=$ 0 , which indicates Cold method measurements when the tuner is initialized and the noise source, toggles from Hot to Cold (Step 2 in Section II-B). For example, ambient temperature $T_{a}$ measured for each tuner setting $i$ will be labeled as $T_{a, i}$ and $T_{a}$ measured in Step 2 in Section II-B will appear as $T_{a, 0}$.

- $B$ : Noise bandwidth.

- $E$ : ENR of the noise source defined in (1).

- $E_{\text {rec }}^{\prime}$ : Effective ENR of the receiver driven by the tuner [see (15) and (25)].

- $E_{\text {sys }}^{\prime}$ : Effective ENR of the system, defined in (31).

- $F_{\mathrm{rec}}, F_{\mathrm{DUT}}$ : Receiver and DUT noise factors, respectively.

- $F_{\text {rec }}^{c(h)}$ : Receiver noise factors when driven by the InputNet.

- $F_{\min }$ : Minimum noise factor.

- $\Gamma_{\mathrm{ns}}^{c(h)}$ : Cold (Hot) noise source reflection coefficients.

- $\Gamma_{\text {rec }} \Gamma_{i \mathrm{Net}}^{c(h)}, \Gamma_{\text {out }}^{c(h)}$ : Reflection coefficients at the InputNet output and at the DUT output

$$
\begin{aligned}
& \Gamma_{i \mathrm{Net}}^{c(h)}=S_{22}^{A}+\frac{S_{21}^{A} S_{12}^{A} \Gamma_{\mathrm{ns}}^{c(h)}}{1-S_{11}^{A} \Gamma_{\mathrm{ns}}^{c(h)}} \\
& \Gamma_{\mathrm{out}}^{c(h)}=S_{22}^{\mathrm{DUT}}+\frac{S_{21}^{\mathrm{DUT}} S_{12}^{\mathrm{DUT}} \Gamma_{i \mathrm{Net}}^{c(h)}}{1-S_{11}^{\mathrm{DUT}} \Gamma_{i \mathrm{Net}}^{c(h)}} .
\end{aligned}
$$

- $\Gamma_{L}^{\text {rec: }}$ Reflection coefficient of the internal receiver load.

- $\Gamma_{\text {opt }}$ : Optimum signal-source reflection coefficient for minimum noise.

- $\Gamma_{g}$ : Generator output reflection coefficient.

- $G_{A, i \text { Net }}^{c(h)}, G_{A}^{\mathrm{DUT}, c(h)}$ : Available gains, relating the available power at the input to the available power at the output, of InputNet, and InputNet followed by the DUT

$$
\begin{aligned}
G_{A, i \mathrm{Net}}^{c(h)} & =\frac{1-\left|\Gamma_{\mathrm{ns}}^{c(h)}\right|^{2}}{\left|1-\Gamma_{\mathrm{ns}}^{c(h)} S_{11}^{A}\right|^{2}} \frac{\left|S_{21}^{A}\right|^{2}}{1-\left|\Gamma_{i \mathrm{Net}}^{c(h)}\right|^{2}} \\
G_{A}^{\mathrm{DUT}, c(h)} & =\frac{1-\left|\Gamma_{i \mathrm{Net}}^{c(h)}\right|^{2}}{\left|1-\Gamma_{i \mathrm{Net}}^{c(h)} S_{11}^{\mathrm{DUT}}\right|^{2}} \frac{\left|S_{21}^{\mathrm{DUT}}\right|^{2}}{1-\left|\Gamma_{\mathrm{out}}^{c(h)}\right|^{2}} .
\end{aligned}
$$

- $G_{T, \text { rec }}^{c(h)}$ : Receiver transducer Cold (Hot) gains, relating the available power at the input of the receiver to the delivered power to the receiver internal load, defined in (5).

- $G_{\text {rec}}$ : Part of the receiver transducer gain that is due to internal response of the receiver, defined in (5).

- $k$ : Boltzmann's constant.

- $M^{c(h)}, M$ : Receiver input mismatch factors when driven by the tuner in (5) and their ratio $M \equiv M^{c} / M^{h}$, respectively.

- $M_{\mathrm{DUT}}^{c(h)}$ : Receiver input mismatch factors when driven by the DUT, defined in (B.8).

- $N$ : Number of tuner settings, or equivalently, number of points in impedance constellations.

- $P_{\mathrm{DUT}}^{c(h)}$ : Input-referred DUT noise powers when driven by InputNet, defined in (B.7).

- $P_{\text {in }}^{c(h)}$ : Available noise powers from the noise source and InputNet, defined in (6).

- $P_{\text {meas }}^{c(h)}, P_{\text {cal }}^{c(h)}$ : Noise power reported by the receiver with and without the DUT, respectively, defined in (4) and (B.6).

- $P_{\text {rec,cal }}^{c(h)}, P_{\text {rec,meas }}^{c(h)}$ : Input-referred noise powers of the receiver driven by the tuner and by the DUT at $T_{0}$, defined in (4) and (19), respectively.

- $R_{n}$ : Equivalent noise resistance.

- $\left[S^{\mathrm{DUT}}\right],\left[S^{i \mathrm{Net}}\right],\left[S^{\mathrm{rec}}\right]$ : DUT, InputNet, and receiver internal $S$-parameters.

- $T_{\text {cal }}^{c(h)}, T_{\text {meas }}^{c(h)}$ : Measured effective system noise temperatures referred to the DUT plane for the calibration steps and the measurement steps, respectively.

- $T_{\mathrm{DUT}}$ : Effective input noise temperatures of the DUT.

- $T_{\text {in }}^{c(h)}$ : Total noise temperatures at the InputNet output when the noise source is OFF (ON), defined in (6).

- $T_{i \text { Net }}^{c(h)}$ : Noise temperatures of InputNet referred to its input, defined in (B.5).

- $T_{\mathrm{ns}}^{c(h)}$ : Cold (Hot) noise source temperatures.

- $T_{\text {rec,cal }}^{c(h)}, T_{\text {rec,meas: Effective DUT-plane referred input }}^{c(h)}$ noise temperatures of the receiver driven by InputNet and by the DUT, respectively.

- $T_{0}, T_{a}^{c(h)} Y_{\text {rec }}, Y_{\text {sys }}$ : Receiver $Y$-factor, defined in (22), and system $Y$-factor, defined in (28), respectively.

- $Y_{\text {rec }}^{\prime}$ : Receiver effective $Y$-factor, defined in (16) and (26).

- $Y_{\text {sys }}^{\prime \prime}, Y_{\text {sys }}^{\prime}:$ System effective $Y$-factors scaled and not scaled by the DUT's available gain ratio, respectively, defined in (30).

- $Z_{0}$ : Characteristic impedance.

\section{APPENDIX II \\ OTHER EXPRESSIONS USED IN THIS STUDY \\ A. Noise Temperature of InputNet}

In (6), $T_{i \text { Net }}^{c(h)}$ are found by replacing the noise source with terminations at ambient temperature $T_{a}^{c(h)}$ with $\Gamma_{\mathrm{ns}}^{c(h)}$ and realizing that since InputNet is a passive device, the noise temperature at its output equals to $T_{a}^{c(h)}$. This expressed as $\left(T_{a}^{c(h)}+\right.$ $\left.T_{i \mathrm{Net}}^{c(h)}\right) G_{A, i \mathrm{Net}}^{c(h)}=T_{a}^{c(h)}$ gives

$$
T_{i \mathrm{Net}}^{c(h)}=T_{a}^{c(h)}\left(\frac{1}{G_{A, i \mathrm{Net}}^{c(h)}}-1\right) .
$$


B. Receiver Reported Noise Temperature During Cold Method DUT Measurements

Modifying (7) to express the noise powers of the system as reported by the receiver gives

$$
\begin{aligned}
P_{\text {meas }, i}^{c}=\left[\left(P_{\mathrm{in}, i}^{c}+\frac{T_{a, i}^{c}}{T_{0}} P_{\mathrm{DUT}, i}^{c}\right) G_{A, i}^{\mathrm{DUT}, c}\right. \\
\\
\left.+\frac{T_{a, i}^{c}}{T_{0}} P_{\text {rec,meas }, i}^{c}\right] M_{\mathrm{DUT}, i}^{c} G_{\text {rec }}
\end{aligned}
$$

where $1 \leq i \leq N, P_{\mathrm{in}, i}^{c}$ is found from (6), $P_{\mathrm{rec}, \text { meas }, i}$ are calculated based on the noise parameters obtained during the receiver calibration and they represent the receiver-input referred noise power when the receiver sees the DUT output reflection coefficient $\Gamma_{\text {out }, i}^{c}$ found from (A.2). $G_{A, i}^{\mathrm{DUT}, c}$ is the DUT available gain (A.4), $P_{\mathrm{DUT}, i}^{c}$ is the noise power of the DUT referenced to the DUT input at $T_{0}$ and

$$
P_{\mathrm{DUT}, i}^{c}=k B T_{\mathrm{DUT}, i}
$$

where $T_{\mathrm{DUT}, i}$ is the effective input noise temperature of the DUT, and is similar to (4)

$$
M_{\mathrm{DUT}, i}^{c} \equiv \frac{1-\left|\Gamma_{\mathrm{out}, i}^{\mathrm{DUT}, c}\right|^{2}}{\left|1-\Gamma_{\mathrm{out}, i}^{\mathrm{DUT}, c} \Gamma_{\mathrm{rec}}\right|^{2}} .
$$

\section{Modified Y-Factor Method: Receiver Y-Factor}

From (21), the $Y$-factor defined as a ratio of measured powers is

$$
\begin{aligned}
Y_{\mathrm{rec}, i} & =\frac{P_{\mathrm{cal}, i}^{h}}{P_{\mathrm{cal}, i}^{c}} \\
& =\frac{P_{\mathrm{in}, i}^{h}+\frac{T_{a, i}^{h}}{T_{0}} P_{\mathrm{rec}, \mathrm{cal}, i}^{h}}{P_{\mathrm{in}, i}^{c}+\frac{T_{a, i}^{c}}{T_{0}} P_{\mathrm{rec}, \mathrm{cal}, i}^{c}} \frac{G_{T, \mathrm{rec}}^{h}}{G_{T, \mathrm{rec}}^{c}} \\
& =\frac{T_{\mathrm{in}, i}^{h}+\frac{T_{a, i}^{h}}{T_{0}} T_{\mathrm{rec}, \mathrm{cal}, i}^{h}}{T_{\mathrm{in}, i}^{c}+\frac{T_{a, i}^{c}}{T_{0}} T_{\mathrm{rec}, \mathrm{cal}, i}^{c}} \frac{G_{T, \mathrm{rec}}^{h}}{G_{T, \mathrm{rec}}^{c}}
\end{aligned}
$$

where $T_{\mathrm{rec}, \mathrm{cal}, i}^{c(i)}$ are receiver effective input noise temperatures when ambient temperature is $290 \mathrm{~K}$ and they relate to receiverinput referred receiver noise power by $P_{\mathrm{rec}, \mathrm{cal}, i}^{c(h)}=k T_{\mathrm{rec}, \mathrm{cal}, i}^{c(h)} B$, and $T_{\mathrm{in}, i}^{c(h)}$ obtained using (6).

\section{Modified Y-Factor Method: DUT Measurement}

For each impedance presented by the tuner to the DUT input, the receiver reported noise powers $P_{\text {meas }, i}^{c(h)}$ are used to find the system $Y$-factor (B.10)

$Y_{\text {sys }, i}$

$$
\begin{aligned}
\equiv & \frac{P_{\text {meas }, i}^{h}}{P_{\text {meas }, i}^{c}} \\
= & \frac{G_{T, \mathrm{rec}, i}^{h}}{G_{T, \mathrm{rec}, i}^{c}} \\
& \times \frac{\left[P_{\mathrm{in}, i}^{h} G_{A, i \mathrm{Net}}^{c h}+\frac{T_{a, i}^{h}}{T_{0}} P_{\mathrm{DUT}, i}^{h}\right] G_{A, i}^{\mathrm{DUT}, h}+\frac{T_{a, i}^{h}}{T_{0}} P_{\mathrm{rec}, \text { meas }, i}^{h}}{\left[P_{\mathrm{in}, i}^{c} G_{A, i \mathrm{Net}}^{c}+\frac{T_{a, i}^{c}}{T_{0}} P_{\mathrm{DUT}, i}^{c}\right] G_{A, i}^{\mathrm{DUT}, c}+\frac{T_{a, i}^{c}}{T_{0}} P_{\mathrm{rec}, \text { meas }, i}^{c}}
\end{aligned}
$$

where receiver-input referred noise powers $P_{\text {rec,meas }, i}^{c(h)}$ when driven by the DUTs are obtained from the noise parameters determined during the receiver calibration, and $G_{A, i}^{\mathrm{DUT}, c(h)}$ are the DUT available gains as in (A.4). In (28), the unknown $P_{\mathrm{DUT}, i}^{c(h)}$ are related through DUT noise parameters.

\section{REFERENCES}

[1] V. Adamian and A. J. Uhlir, "A novel procedure for receiver noise characterization," IEEE Trans. Instrum. Meas., vol. IM-22, no. 2, pp. 181-182, Jun. 1973.

[2] A. C. Davidson, B. W. Leake, and E. Strid, "Accuracy improvements in microwave noise parameter measurements," IEEE Trans. Microw. Theory Tech., vol. 37, no. 12, pp. 1973-1978, Dec. 1989.

[3] M. N. Tutt, "Low and high frequency noise properties of heterojunction transistors," Ph.D. dissertation, Dept. Elect. Eng. Comput. Sci., The University of Michigan at Ann Arbor, Ann Arbor, MI, 1994.

[4] A. Lzaro, L. Pradell, and J. O'Callaghan, "Method for measuring noise parameters of microwave two-port," Electron. Lett., vol. 34, no. 13, pp. 1332-1333, Jun. 1998.

[5] P. Bland, L. Roy, S. Labont, and M. Stubbs, "An enhanced on-wafer millimeter-wave noise parameter measurement system," IEEE Trans. Instrum. Meas., vol. 48, no. 4, pp. 825-829, Aug. 1999.

[6] T. Vähä-Heikkilä, M. Lahdes, M. Kantanen, and J. Tuovinen, "Onwafer noise-parameter measurements at $W$-band," IEEE Trans. Microw. Theory Tech., vol. 51, no. 6, pp. 1621-1628, Jun. 2003.

[7] J. Randa and D. Walker, "On-wafer measurement of transistor noise parameters at NIST," IEEE Trans. Instrum. Meas., vol. 56, no. 2, pp. 551-554, Apr. 2007.

[8] R. Meierer and C. Tsironis, "An on-wafer noise paramenter measurement technique with automatic receiver calibration," Microw. J., vol. 38, no. 3, pp. 22-37, Mar. 1995.

[9] G. Caruso and M. Sannino, "Determination of microwave two-port noise parameters through computer-aided frequency-conversion techniques," IEEE Trans. Microw. Theory Tech., vol. MTT-27, no. 9, pp. 779-783, Sep. 1979.

[10] J. Archer and R. Batchelor, "Fully automated on-wafer noise characterization of GaAs MESFETs and HEMTs," IEEE Trans. Microw. Theory Tech., vol. 40, no. 2, pp. 209-216, Feb. 1992.

[11] L. F. Tiemeijer, R. J. Havens, R. de Kort, and A. J. Scholten, "Improved $Y$-factor method for wide-band on-wafer noise-parameter measurements," IEEE Trans. Microw. Theory Tech., vol. 53, no. 9, pp. 2917-2925, Sep. 2005.

[12] R. Meys, "A wave approach to the noise properties of linear microwave devices," IEEE Trans. Microw. Theory Tech., vol. MTT-26, no. 1, pp. 34-37, Jan. 1978.

[13] S. Withington, "Scattered noise waves in microwave and mm-wave networks," Microw. J., vol. 32, no. 6, pp. 169-178, Jun. 1989.

[14] S. Wedge and D. Rutledge, "Wave techniques for noise modeling and measurement," IEEE Trans. Microw. Theory Tech., vol. 40, no. 11, pp. 2004-2012, Nov. 1992.

[15] T. Werling, E. Bourdel, D. Pasquet, and A. Boudiaf, "Determination of wave noise sources using spectral parametric modeling," IEEE Trans. Microw. Theory Tech., vol. 45, no. 12, pp. 2461-2467, Dec. 1997.

[16] V. Danelon, P. Corzat, F. Aniel, and G. Vernet, "Four noise parameter determination method for transistors based on the frequency dependence of the noise figure," Electron. Lett., vol. 34, no. 16, pp. 1612-1613, Aug. 1998.

[17] R. Hu and S. Weinreb, "A novel wide-band noise-parameter measurement method and its cryogenic application," IEEE Trans. Microw. Theory Tech., vol. 52, no. 5, pp. 1498-1507, May 2004.

[18] F. Giannini, E. Bourdel, and D. Pasquet, "A new method to extract noise parameters based on a frequency- and time-domain analysis of noise power measurements," IEEE Trans. Instrum. Meas., vol. 57, no. 2, pp. 261-267, Feb. 2008.

[19] G. Dambrine, H. Happy, F. Danneville, and A. Cappy, "A new method for on wafer noise measurement," IEEE Trans. Microw. Theory Tech., vol. 41, no. 3, pp. 375-381, Mar. 1993.

[20] A. Làzaro, L. Pradell, and J. O'Callaghan, "FET noise-parameter determination using a novel technique based on $50-\Omega$ noise-figure measurements," IEEE Trans. Microw. Theory Tech., vol. 47, no. 3, pp. 315-324, Mar. 1999. 
[21] S. Asgaran, M. J. Deen, C.-H. Chen, G. A. Rezvani, Y. Kamali, and Y. Kiyota, "Analytical determination of MOSFET's high-frequency noise parameters from $\mathrm{NF}_{50}$ measurements and its application in RFIC design," IEEE J. Solid-State Circuits, vol. 42, no. 5, pp. 1034-1043, May 2007.

[22] P. J. Hall, "The Square Kilometre Array: An international engineering perspective," Experimental Astron., vol. 17, no. 1-3, pp. 5-16, Jun. 2004.

[23] R. Schilizzi, "The square kilometer array," in Proc. SPIE-Int. Soc. Opt. Eng., Glasgow, U.K., Jun. 21-24, 2005, vol. 5489, pp. 62-71, ser. Ground-based telescopes.

[24] P. Dewdney, P. Hall, R. Schilizzi, and T. Lazio, "The Square Kilometre Array," Proc. IEEE, vol. 97, no. 8, pp. 1482-1496, Aug. 2009.

[25] G. Gramegna, M. Paparo, P. G. Erratico, and P. De Vita, "A sub-1-dB $\mathrm{NF}+$ or - 2.3-kV ESD-protected 900-MHz CMOS LNA," IEEE J. Solid-State Circuits, vol. 36, no. 7, pp. 1010-1017, Jul. 2001.

[26] P. Leroux, J. Janssens, and M. Steyaert, "A 0.8-dB NF ESD-protected 9-mW CMOS LNA operating at $1.23 \mathrm{GHz}$ [for GPS receiver]," IEEE J. Solid-State Circuits, vol. 37, no. 6, pp. 760-765, Jun. 2002.

[27] D. J. Cassan and J. R. Long, "A 1-V transformer-feedback low-noise amplifier for 5-GHz wireless LAN in 0.18- $\mu \mathrm{m}$ CMOS," IEEE J. SolidState Circuits, vol. 38, no. 3, pp. 427-435, Mar. 2003.

[28] J. Xu, B. Woestenburg, J. G. Bij de Vaate, and W. A. Serdijn, "GaAs $0.5 \mathrm{~dB}$ NF dual-loop negative-feedback broadband low-noise amplifier IC," Electron. Lett., vol. 41, no. 14, pp. 780-782, Jul. 2005.

[29] E. A. M. Klumperink, Q. Zhang, G. J. M. Wienk, R. Witvers, J. G. Bij de Vaate, B. Woestenburg, and B. Nauta, "Achieving wideband sub-1 $\mathrm{dB}$ noise figure and high gain with MOSFETs if input power matching is not required," in IEEE Radio Freq. Integr. Circuits Symp., Honolulu, HI, Jun. 3-5, 2007, pp. 673-676.

[30] S. Weinreb, J. Bardin, and H. Mani, "Design of cryogenic SiGe lownoise amplifiers," IEEE Trans. Microw. Theory Tech., vol. 55, no. 11, pp. 2306-2312, Nov. 2007.

[31] L. Belostotski and J. W. Haslett, "Wide band room temperature 0.35-dB noise figure LNA in 90-nm bulk CMOS," in IEEE Radio Wireless Symp., Long Beach, CA, Jan. 7-11, 2007, pp. 221-224.

[32] L. Belostotski and J. W. Haslett, "Noise figure optimization of wide-band inductively-degenerated CMOS LNAs," in IEEE Midwest Circuits Syst. Symp., Montreal, QC, Canada, Aug. 5-8, 2007, pp. 1002-1005.

[33] L. Belostotski and J. W. Haslett, "Sub-0.2 dB noise figure wide-band room-temperature CMOS LNA with non-50 $\Omega$ signal-source impedance," IEEE J. Solid-State Circuits, vol. 42, no. 11, pp. 2492-2502, Nov. 2007.

[34] L. Belostotski and J. W. Haslett, "Two-port noise figure optimization of source-degenerated cascode CMOS LNAs," Analog Integr. Circuits Signal Process., vol. 55, no. 2, pp. 125-137, May 2008.

[35] L. Belostotski and J. W. Haslett, "A technique for differential noise figure measurement of differential LNAs," IEEE Trans. Instrum. Meas., vol. 57, no. 7, pp. 1298-1303, Jul. 2008.

[36] "Agilent fundamentals of RF and microwave noise figure measurements," Agilent Technol., Santa Clara, CA, Appl. Note 57-1, Oct. 2006.

[37] "Noise figure measurement accuracy-The $Y$-factor method," Agilent Technol., Santa Clara, CA, Appl. Note 57-2, Oct. 2004

[38] H. T. Friis, "Noise figures of radio receivers," Proc. IRE, vol. 32, no. 7, pp. 419-422, Jul. 1944.

[39] A. van der Ziel, "Thermal noise in field-effect transistors," Proc. IRE, vol. 50, no. 8, pp. 1808-1812, Aug. 1962.

[40] A. van der Ziel, "The state of solid state device noise research," in 4th Int. Phys. Aspects of Noise in Solid State Devices Conf., Noordwij, The Netherlands, May 1976, pp. 41-51.

[41] A. van der Ziel, Noise in Solid State Devices and Circuits. New York: Wiley, 1986.

[42] J. W. Haslett and F. N. Trofimenkoff, "Thermal noise in field-effect devices," Proc. IEEE, vol. 116, no. 11, pp. 1863-1868, Nov. 1969.

[43] F. N. Trofimenkoff, J. W. Haslett, and R. E. Smallwood, "Hot electron thermal noise models for FETs," Int. J. Electron., vol. 44, no. 3, pp. 257-272, Mar. 1978.

[44] "IRE standards on methods of measuring noise in linear twoports, 1959," Proc. IRE, vol. 48, no. 1, pp. 60-68, Jan. 1960.

[45] G. Gonzalez, Microwave Transistor Amplifiers: Analysis and Design, 2nd ed. Toronto, ON, Canada: Prentice-Hall, 1997.

[46] R. Lane, "The determination of device noise parameters," Proc. IEEE, vol. 57, no. 8, pp. 1461-1462, Aug. 1969

[47] M. Mitama and H. Katoh, "An improved computational method for noise parameter measurement," IEEE Trans. Microw. Theory Tech., vol. MTT-27, no. 6, pp. 612-615, Jun. 1979.
[48] G. Vasilescu, G. Alquie, and M. Krim, "Exact computation of two-port noise parameters," Electron. Lett., vol. 25, no. 4, pp. 292-293, Feb. 1989.

[49] A. Boudiaf and M. Laporte, "An accurate and repeatable technique for noise parameter measurements," IEEE Trans. Instrum. Meas., vol. 42, no. 2, pp. 532-537, Nov. 1993.

[50] C.-H. Chen, Y.-L. Wang, M. H. Bakr, and Z. Zeng, "Novel noise parameter determination for on-wafer microwave noise measurements," IEEE Trans. Instrum. Meas., vol. 57, no. 11, pp. 2462-2471, Nov. 2008.

[51] M. Sannino, "On the determination of device noise and gain parameters," Proc. IEEE, vol. 67, no. 9, pp. 1364-1366, Sep. 1979.

[52] J. O'Callaghan and J. Mondal, "A vector approach for noise parameter fitting and selection of source admittances," IEEE Trans. Microw. Theory Tech., vol. 39, no. 8, pp. 1376-1382, Aug. 1991.

[53] S. Van den Bosch and L. Martens, "Improved impedance-pattern generation for automatic noise-parameter determination," IEEE Trans. Microw. Theory Tech., vol. 46, no. 11, pp. 1673-1678, Nov. 1998.

[54] G. Banerjee, K. Soumyanath, and D. Allstot, "Measurement and modeling errors in noise parameters of scaled-CMOS devices," IEEE Trans. Microw. Theory Tech., vol. 54, no. 6, pp. 2336-2345, Jun. 2006.

[55] J. Randa and W. Wiatr, "Monte carlo estimation of noise-parameter uncertainties," Proc. Inst. Elect. Eng.-Sci., Meas., Technol., vol. 149, no. 6, pp. 333-337, Nov. 2002

[56] J. Randa, "Noise-parameter uncertainties: A Monte Carlo simulation," J. Res. NIST, vol. 107, no. 5, pp. 431-444, Sep.-Oct. 2002.

[57] L. Escotte, R. Plana, and J. Graffeuil, "Evaluation of noise parameter extraction methods," IEEE Trans. Microw. Theory Tech., vol. 41, no. 3, pp. 382-387, Mar. 1993.

[58] A. Boudiaf and M. Laporte, "Evaluation des méthodes d'extraction des paramtères de bruit," presented at the IEEE French Chapter Meeting, Carcassonne, France, Nov. 22-23, 1990.

[59] A. Boudiaf, M. LaPorte, J. Dangla, and G. Vernet, "Accuracy improvements in two-port noise parameter extraction method," in IEEE MTT-S Int. Microw. Symp. Dig., Albuquerque, NM, Jun. 1-5, 1992, pp. $1569-1572$

[60] D. K. Rytting, "Network analyzer accuracy overview," in Proc. 58th ARFTG Conf., 2001, vol. 40, pp. 1-13.

[61] "Tuner repeatability: Fact and fiction," Maury Microwave Corporation, Ontario, CA, Appl. Note 5C-032, Jun. 09, 1999.

[62] J. Sevic, "Introduction to tuner-based measurement and characterization," Maury Microwave Corporation, Ontario, CA, Appl. Note 5C-054, Aug. 31, 2004.

[63] "Long and short term tuner accuracy and calibration repeatability," Focus Microw. Inc., Saint-Laurent, QC, Canada, Product Note 50, 1999.

[64] "Smart Noise Sources SNS Series: Operating and Service Guide (N4000A, N4001A, and N4002A)," Agilent Technol., Palo Alto, CA, 2001.

[65] "Agilent N8973A, N8974A, N8975A NFA series noise figure analyzers: Data sheet," Agilent Technol., Palo Alto, CA, 2007.

[66] J. Randa, "Uncertainties in NIST noise-temperature measurements," NIST, Boulder, CO, Tech. Rep. 1502, 1998.

[67] W. Wiatr and D. Walker, "Systematic errors of noise parameter determination caused by imperfect source impedance measurement," IEEE Trans. Instrum. Meas., vol. 54, no. 2, pp. 696-700, Apr. 2005.

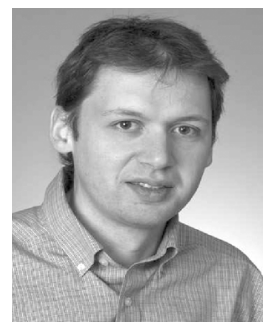

Leonid Belostotski (S'97-M'01) received the B.Sc. and M.Sc. degrees in electrical engineering from the University of Alberta, Edmonton, AB, Canada, in 1997 and 2000, respectively, and the Ph.D. degree from the University of Calgary, Calgary, $\mathrm{AB}$, Canada, in 2007.

A large portion of his M.Sc. thesis program was spent with the Dominion Radio Astrophysical Observatory, NRC, Penticton, BC, Canada, where he designed and prototyped a distance measurement and phase synchronization system for the Canadian large adaptive reflector telescope. Following his graduation, he was an RF Engineer with Murandi Communications Ltd., during which time he designed devices for high-volume consumer applications and low-volume high-performance devices for the James Clerk Maxwell telescope in Honolulu, HI. In 2007, he became an Assistant Professor with the University of Calgary. His doctoral research concerned low-noise amplifiers for the SKA radio telescope. 


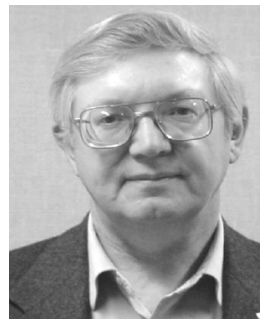

James W. Haslett (S'64-M'66-SM'79-F'02) is currently the Director of the provincial iCORE-funded Advanced Technology Information Processing Systems (ATIPS) Laboratory, University of Calgary, Calgary, AB, Canada. In July 2007, he became a University Professor with the University of Calgary. From 1986 to 1997, he was Head of the Department of Electrical Engineering, University of Calgary. He has been President of his own engineering consulting firm since 1981, consulting to oil-field instrumentation firms primarily on high-temperature and downhole instrumentation. He was also a member of several national and international science teams designing satellite instrumentation in the late 1970s and 1980s. He held the TRLabs/iCORE/NSERC Senior Industrial Research
Chair in Wireless Communications from 2002 to 2007, building a team of researchers specializing in RF integrated circuit (RFIC) design for wireless communications applications. He has authored or coauthored over 200 papers in peer-reviewed journals and conference proceedings. He holds 12 patents, many of which have been licensed to industry. He has graduated over 40 M.Sc. and Ph.D. students during his career.

Dr. Haslett is a Fellow of the Engineering Institute of Canada and the Canadian Academy of Engineering. He is currently a member of the Editorial Review Committees of five IEEE TRANSACTIONS. He is a member of several technical and executive committees of international IEEE conferences and is also a member of the provincial iCORE Internal Review Committee that establishes research chair programs in Alberta, Canada. He and his students have been the recipients of numerous national and international awards for their research. 